\title{
Weighted pseudo-almost periodic solutions and global exponential synchronization for delayed QVCNNs
}

\author{
Yongkun Li' ${ }^{*}$ (D), Gui Lü ${ }^{1}$ and Xiaofang Meng ${ }^{1}$
}

"Correspondence: yklie@ynu.edu.cn

'Department of Mathematics, Yunnan University, Kunming,

People's Republic of China

\section{Springer}

\begin{abstract}
In this paper, we study the existence of weighted pseudo-almost periodic solutions and the global exponential synchronization of delayed quaternion-valued cellular neural networks (QVCNNs). Firstly, we use the Banach fixed point theorem to establish the existence of weighted pseudo-almost periodic solutions for this class of QVCNNs. Then, under the condition that the drive system has a unique weighted pseudo-almost periodic solution, by designing a state-feedback controller and constructing suitable Lyapunov functions, we see that the drive-response structure of delayed QVCNNs with weighted pseudo-almost periodic coefficients achieve global exponential synchronization. Finally, a numerical example is given to illustrate the feasibility of our results.
\end{abstract}

MSC: 34K14; 34D06; 92B20

Keywords: Weighted pseudo-almost periodic solution; Synchronization; Quaternion; Cellular neural networks

\section{Introduction}

Cellular neural networks (CNNs), which were originally proposed by Chua and Yang in [1, 2], have been widely used in signal processing, pattern recognition, associative memory, combinatorial optimization, intelligent robot control, and other new fields of application are constantly being discovered. In the past 30 years, many authors have considered the existence, uniqueness and stability of equilibrium points ([3]), periodic solutions $([4,5])$, almost periodic solutions ([6, 7]), pseudo-almost periodic solutions $([8,9])$ and weighted pseudo-almost periodic solutions $([10,11])$ of CNNs. In addition, as is well known, for artificial neural network systems and theoretical ecosystems, the dynamic behavior of the systems is the focus of great concern and interest. Stability, periodicity and almost periodicity are important dynamic characteristics of the systems. Therefore, these behaviors of neural network systems and ecosystems have been extensively studied (see [12-24]). In addition, we know that weighted pseudo-almost periodicity is an extension of pseudoalmost periodicity and pseudo-almost periodicity. However, to the best of our knowledge, the results of weighted pseudo-almost periodic solutions for CNNs are still rare.

On the one hand, synchronization is a common phenomenon in real world systems. This means that two or more systems are mutually regulated to reach a common dynamic be-

(c) The Author(s) 2019. This article is distributed under the terms of the Creative Commons Attribution 4.0 International License (http://creativecommons.org/licenses/by/4.0/), which permits unrestricted use, distribution, and reproduction in any medium, provided you give appropriate credit to the original author(s) and the source, provide a link to the Creative Commons license, and indicate if changes were made. 
havior. Since Pecora and Carrol in [25] introduced the concept of drive-response synchronization for coupled chaotic systems, chaos synchronization has become a hot research topic due to its potential applications in secure communication, automatic control, biological systems, information science $([26,27])$. Also, the synchronization of neural networks has been the focus of scientific research and has been widely studied (see [28-34]).

On the other hand, it is well known that a quaternion consists of a real and three imaginary parts [35]. The three imaginary units $i, j$ and $k$ obey Hamilton's multiplication rules:

$$
i j=-j i=k, \quad j k=-k j=i, \quad k i=-i k=j, \quad i^{2}=j^{2}=k^{2}=i j k=-1 .
$$

The skew field of a quaternion is denoted by $\mathbb{H}:=\left\{h=h^{R}+i h^{I}+j h^{J}+k h^{K}\right\}$, where $h^{R}, h^{I}, h^{J}, h^{K} \in \mathbb{R}$.

In recent years, quaternion-valued neural networks, which can be seen as a generic extension of complex-valued neural networks or real-valued neural networks, have been found many practical applications and have been widely concerned [36, 37]. Since the application of neural networks depends on their dynamics, some papers have been devoted to the study of the dynamical behaviors for quaternion-valued neural networks ([38-43]). However, up to now, there are still no results about weighted pseudo-almost periodic solutions and synchronization of QVCNNs. Therefore, it is very important and necessary to study the weighted pseudo-almost periodicity and synchronization of QVCNNs.

Motivated by the above discussion, in this paper, we consider the following delayed QVCNN:

$$
x_{p}^{\prime}(t)=-c_{p}(t) x_{p}(t)+\sum_{q=1}^{n} a_{p q}(t) f_{q}\left(x_{q}(t)\right)+\sum_{q=1}^{n} b_{p q}(t) g_{q}\left(x_{q}\left(t-\tau_{p q}(t)\right)\right)+J_{p}(t),
$$

where $p \in\{1,2, \ldots, n\}:=S, n$ corresponds to the number of units in the neural network; $x_{p}(t)$ is the state of the $p$ th neuron at time $t ; c_{p}(t)>0$ is the self-feedback connection weight; $a_{p q}(t), b_{p q}(t)$ represent the connection weight and the delay connection weight between cell $p$ and $q$ at time $t$, respectively; $J_{p}(t)$ is an external input on the $p$ th unit at time $t ; f_{q}$ and $g_{q}$ are activation functions; $\tau_{p q}(t)$ represents the transmission delay at time $t$.

The initial value is given by

$$
x_{p}(s)=\varphi_{p}(s), \quad s \in[-\tau, 0], p \in S,
$$

where $\tau=\max _{p, q \in S}\left\{\sup _{t \in \mathbb{R}}\left|\tau_{p q}(t)\right|\right\}, \varphi_{p}(s)=\varphi_{p}^{R}(s)+i \varphi_{p}^{I}(s)+j \varphi_{p}^{J}(s)+k \varphi_{p}^{K}(s)$ is a continuous function.

This paper is organized as follows: In Sect. 2, we introduce some definitions, preliminary lemmas. In Sect. 3, we establish some sufficient conditions for the existence of weighted pseudo-almost periodic solutions of (1). In Sect. 4, global exponential synchronization is investigated. In Sect. 5, we give an example to demonstrate the feasibility of our results. This paper ends with a brief conclusion in Sect. 6 .

\section{Preliminaries}

Let $B C\left(\mathbb{R}, \mathbb{R}^{n}\right)$ be the set of all bounded and continuous functions from $\mathbb{R}$ to $\mathbb{R}^{n}$.

Definition $1([44,45])$ A function $f \in B C\left(\mathbb{R}, \mathbb{R}^{n}\right)$ is said to be almost periodic if, for any $\epsilon>0$, it is possible to find a real number $l=l(\epsilon)>0$, for any interval with length $l(\epsilon)$, there 
exists a number $\tau=\tau(\epsilon)$ in this interval such that $|f(t+\tau)-f(t)|<\epsilon$ for all $t \in \mathbb{R}$. The collection of such functions will be denoted by $A P\left(\mathbb{R}, \mathbb{R}^{n}\right)$.

Let $\mathbb{W}$ denote the collection of functions (weights) $v: \mathbb{R} \rightarrow(0,+\infty)$, which are locally integrable over $\mathbb{R}$ such that $v>0$ almost everywhere. If $v \in \mathbb{W}$ and for $r>0$, we set $Q_{r}:=$ $[-r, r]$ and

$$
v\left(Q_{r}\right):=\int_{Q_{r}} v(x) \mathrm{d} x .
$$

Let

$$
\mathbb{W}_{\infty}=\left\{v \in \mathbb{W}: \inf _{x \in \mathbb{R}} v(x)=v_{0}>0, \lim _{r \rightarrow \infty} v\left(Q_{r}\right)=\infty\right\} .
$$

Definition 2 ([46]) Fix $v \in \mathbb{W}_{\infty}$. A continuous function $f \in B C(\mathbb{R}, \mathbb{X})$ is called weighted pseudo-almost periodic if it can be written as $f=g+h$ with $g \in A P(\mathbb{R}, \mathbb{X})$ and $h \in$ $P A P_{0}(\mathbb{R}, \mathbb{X}, v)$, where the space $P A P_{0}(\mathbb{R}, \mathbb{X}, v)$ is defined by

$$
P A P_{0}(\mathbb{R}, \mathbb{X}, v)=\left\{g \in B C(\mathbb{R}, \mathbb{X}): \lim _{r \rightarrow \infty} \frac{1}{v\left(Q_{r}\right)} \int_{Q_{r}}\|g(t)\| v(t) \mathrm{d} t=0\right\} .
$$

The collection of all weighted pseudo-almost periodic functions $f: \mathbb{R} \rightarrow \mathbb{R}^{n}$ will be denoted by $P A P\left(\mathbb{R}, \mathbb{R}^{n}, v\right)$.

Lemma 1 ([47]) If $f, g \in P A P(\mathbb{R}, \mathbb{R}, v)$, then $f+g, f g \in P A P(\mathbb{R}, \mathbb{R}, v)$; if $f \in P A P(\mathbb{R}, \mathbb{R}, v)$, $g \in A P(\mathbb{R}, \mathbb{R})$, then $f g \in P A P(\mathbb{R}, \mathbb{R}, v)$.

Lemma 2 ([47]) Fix $v \in \mathbb{W}_{\infty}$. Suppose that, for any $s \in \mathbb{R}$,

$$
\varlimsup_{|t| \rightarrow \infty} \frac{v(t+s)}{v(t)}<\infty
$$

Then $P A P_{0}(\mathbb{R}, \mathbb{X}, v)$ is translation-invariant.

Denote

$$
\mathbb{W}_{\infty}^{\mathrm{Inv}}=\left\{v \in \mathbb{W}_{\infty}: \forall s \in \mathbb{R}, \varlimsup_{|t| \rightarrow \infty} \frac{v(t+s)}{v(t)}<\infty\right\} .
$$

Lemma 3 ([10]) If $f \in C(\mathbb{R}, \mathbb{R})$ satisfies the Lipschitz condition, $\varphi \in P A P(\mathbb{R}, \mathbb{R}, v)$ and $\delta \in$ $C(\mathbb{R}, \mathbb{R})$, then $f(\varphi(t-\delta(t))) \in \operatorname{PAP}(\mathbb{R}, \mathbb{R}, v)$.

Definition 3 Fix $v \in \mathbb{W}_{\infty}$. Let $f=f^{R}+i f^{I}+j f^{I}+k f^{K}: \mathbb{R} \rightarrow \mathbb{H}$ where $f^{l}: \mathbb{R} \rightarrow \mathbb{R}, l \in$ $\{R, I, J, K\}:=T . f$ is said to be weighted pseudo-almost periodic if, for every $l \in T, f^{l}$ is weighted pseudo-almost periodic. The collection of such functions will be denoted by $\operatorname{PAP}(\mathbb{R}, \mathbb{H}, v)$.

Let $x_{q}=x_{q}^{R}+i x_{q}^{I}+j x_{q}^{I}+k x_{q}^{K} \in \mathbb{H}$, where $x_{q}^{l}: \mathbb{R} \rightarrow \mathbb{R}, l \in T$. Then $f_{q}\left(x_{q}\right)$ and $g_{q}\left(x_{q}\right)$ of (1) can be expressed as

$$
\begin{aligned}
f_{q}\left(x_{q}\right)= & f_{q}^{R}\left(x_{q}^{R}, x_{q}^{I}, x_{q}^{J}, x_{q}^{K}\right)+i f_{q}^{I}\left(x_{q}^{R}, x_{q}^{I}, x_{q}^{J}, x_{q}^{K}\right) \\
& +j f_{q}^{J}\left(x_{q}^{R}, x_{q}^{I}, x_{q}^{J}, x_{q}^{K}\right)+k f_{q}^{K}\left(x_{q}^{R}, x_{q}^{I}, x_{q}^{J}, x_{q}^{K}\right),
\end{aligned}
$$


Li et al. Journal of Inequalities and Applications

(2019) 2019:231

Page 4 of 23

$$
\begin{aligned}
g_{q}\left(x_{q}\right)= & g_{q}^{R}\left(x_{q}^{R}, x_{q}^{I}, x_{q}^{J}, x_{q}^{K}\right)+i g_{q}^{I}\left(x_{q}^{R}, x_{q}^{I}, x_{q}^{J}, x_{q}^{K}\right) \\
& +j g_{q}^{J}\left(x_{q}^{R}, x_{q}^{I}, x_{q}^{J}, x_{q}^{K}\right)+k g_{q}^{K}\left(x_{q}^{R}, x_{q}^{I}, x_{q}^{J}, x_{q}^{K}\right),
\end{aligned}
$$

where $f_{q}^{l}, g_{q}^{l}: \mathbb{R}^{4} \rightarrow \mathbb{R}, q \in S, l \in T$.

According to Hamilton rules, system (1) can be transformed into the following system:

$$
\begin{aligned}
& \left(x_{p}^{R}\right)^{\prime}(t)=-c_{p}(t) x_{p}^{R}(t)+\sum_{q=1}^{n}\left(a_{p q}^{R}(s) f_{q}^{R}\{t, x\}-a_{p q}^{I}(s) f_{q}^{I}\{t, x\}\right. \\
& \left.-a_{p q}^{J}(s) f_{q}^{J}\{t, x\}-a_{p q}^{K}(s) f_{q}^{K}\{t, x\}\right)+\sum_{q=1}^{n}\left(b_{p q}^{R}(s) g_{q}^{R}\{t, \tau, x\}\right. \\
& -b_{p q}^{I}(s) g_{q}^{I}\{t, \tau, x\}-b_{p q}^{J}(s) g_{q}^{J}\{t, \tau, x\} \\
& \left.-b_{p q}^{K}(s) g_{q}^{K}\{t, \tau, x\}\right)+J_{p}^{R}(t) \\
& \triangleq-c_{p}(t) x_{p}^{R}(t)+F_{p}^{R}(t, x(t))+J_{p}^{R}(t), \quad q \in S, \\
& \left(x_{p}^{I}\right)^{\prime}(t)=-c_{p}(t) x_{p}^{I}(t)+\sum_{q=1}^{n}\left(a_{p q}^{R}(s) f_{q}^{I}\{t, x\}+a_{p q}^{I}(s) f_{q}^{R}\{t, x\}\right. \\
& \left.+a_{p q}^{J}(s) f_{q}^{K}\{t, x\}-a_{p q}^{K}(s) f_{q}^{J}\{t, x\}\right)+\sum_{q=1}^{n}\left(b_{p q}^{R}(s) g_{q}^{I}\{t, \tau, x\}\right. \\
& +b_{p q}^{I}(s) g_{q}^{R}\{t, \tau, x\}+b_{p q}^{J}(s) g_{q}^{K}\{t, \tau, x\} \\
& \left.-b_{p q}^{K}(s) g_{q}^{J}\{t, \tau, x\}\right)+J_{p}^{I}(t) \\
& \triangleq-c_{p}(t) x_{p}^{I}(t)+F_{p}^{I}(t, x(t))+J_{p}^{I}(t), \quad q \in S, \\
& \left(x_{p}^{J}\right)^{\prime}(t)=-c_{p}(t) x_{p}^{J}(t)+\sum_{q=1}^{n}\left(a_{p q}^{R}(s) f_{q}^{J}\{t, x\}+a_{p q}^{J}(s) f_{q}^{R}\{t, x\}\right. \\
& \left.-a_{p q}^{I}(s) f_{q}^{K}\{t, x\}+a_{p q}^{K}(s) f_{q}^{I}\{t, x\}\right)+\sum_{q=1}^{n}\left(b_{p q}^{R}(s) g_{q}^{J}\{t, \tau, x\}\right. \\
& +b_{p q}^{J}(s) g_{q}^{R}\{t, \tau, x\}-b_{p q}^{I}(s) g_{q}^{K}\{t, \tau, x\} \\
& \left.+b_{p q}^{K}(s) g_{q}^{I}\{t, \tau, x\}\right)+J_{p}^{J}(t) \\
& \triangleq-c_{p}(t) x_{p}^{J}(t)+F_{p}^{J}(t, x(t))+J_{p}^{J}(t), \quad q \in S, \\
& \left(x_{p}^{K}\right)^{\prime}(t)=-c_{p}(t) x_{p}^{K}(t)+\sum_{q=1}^{n}\left(a_{p q}^{R}(s) f_{q}^{K}\{t, x\}+a_{p q}^{K}(s) f_{q}^{R}\{t, x\}\right. \\
& \left.+a_{p q}^{I}(s) f_{q}^{J}\{t, x\}-a_{p q}^{J}(s) f_{q}^{I}\{t, x\}\right)+\sum_{q=1}^{n}\left(b_{p q}^{R}(s) g_{q}^{K}\{t, \tau, x\}\right. \\
& +b_{p q}^{K}(s) g_{q}^{R}\{t, \tau, x\}+b_{p q}^{I}(s) g_{q}^{J}\{t, \tau, x\} \\
& \left.-b_{p q}^{J}(s) g_{q}^{I}\{t, \tau, x\}\right)+J_{p}^{K}(t) \\
& \triangleq-c_{p}(t) x_{p}^{K}(t)+F_{p}^{K}(t, x(t))+J_{p}^{K}(t), \quad q \in S,
\end{aligned}
$$

where

$$
f_{q}^{l}\{t, x\} \triangleq f_{q}^{l}\left(x_{q}^{R}(t), x_{q}^{I}(t), x_{q}^{J}(t), x_{q}^{K}(t)\right)
$$




$$
g_{q}^{l}\{t, \tau, x\} \triangleq g_{q}^{l}\left(x_{q}^{R}\left(t-\tau_{p q}(t)\right), x_{q}^{I}\left(t-\tau_{p q}(t)\right), x_{q}^{J}\left(t-\tau_{p q}(t)\right), x_{q}^{K}\left(t-\tau_{p q}(t)\right)\right) .
$$

That is, system (1) is decomposed into the following system:

$$
\left(x_{p}^{l}\right)^{\prime}(t)=-c_{p}(t) x_{p}^{l}(t)+F_{p}^{l}(t, x(t))+J_{p}^{l}(t)
$$

where $p \in S, l \in T$. The initial condition associated with (2) is of the form

$$
x_{p}^{l}(s)=\varphi_{p}^{l}(s), \quad s \in[-\tau, 0], p \in S, l \in T .
$$

Remark 1 If $x=\left(x_{1}^{R}, x_{2}^{R}, \ldots x_{n}^{R}, x_{1}^{I}, x_{2}^{I}, \ldots, x_{n}^{I}, x_{1}^{J}, x_{2}^{J}, \ldots, x_{n}^{J}, x_{1}^{K}, x_{2}^{K}, \ldots, x_{n}^{K}\right)^{T}$ is a solution of system (2), then $z=\left(z_{1}, z_{2}, \ldots, z_{n}\right)^{T}$ is a solution of (1), where $z_{p}=x_{p}^{R}+i x_{p}^{I}+j x_{p}^{J}+k x_{p}^{K}, p \in S$, and vice versa.

For the convenience, in the following, we introduce the following notation:

$$
f^{-}=\inf _{t \in \mathbb{R}}|f(t)|, \quad f^{+}=\sup _{t \in \mathbb{R}}|f(t)|,
$$

where $f: \mathbb{R} \rightarrow \mathbb{R}$ is a bounded function.

Throughout the paper, we assume that the following conditions hold:

$\left(H_{1}\right)$ For $p, q \in S, c_{p} \in C\left(\mathbb{R}, \mathbb{R}^{+}\right)$with $c_{p}^{-}=\inf _{t \in \mathbb{R}} c_{p}(t)>0, a_{p q}, b_{p q} \in P A P(\mathbb{R}, \mathbb{H}, \mu), \tau_{p q} \in$ $A P\left(\mathbb{R}, \mathbb{R}^{+}\right)$, for fixed $v \in \mathbb{W}_{\infty}^{\text {Inv }}$, and $J_{p} \in P A P(\mathbb{R}, \mathbb{H})$.

$\left(H_{2}\right)$ Functions $f_{q}^{l}, g_{q}^{l} \in C\left(\mathbb{R}^{4}, \mathbb{R}\right)$ and, for any $x_{q}^{l}, y_{q}^{l} \in \mathbb{R}$, there exist positive constants $L_{f}^{l}$ and $L_{g}^{l}$ such that

$$
\begin{aligned}
& \left|f_{q}^{l}\left(y_{q}^{R}, y_{q}^{I}, y_{q}^{J}, y_{q}^{K}\right)-f_{q}^{l}\left(x_{q}^{R}, x_{q}^{I}, x_{q}^{J}, x_{q}^{K}\right)\right| \\
& \quad \leq L_{f}^{R}\left|y_{q}^{R}-x_{q}^{R}\right|+L_{f}^{I}\left|y_{q}^{I}-x_{q}^{I}\right|+L_{f}^{J}\left|y_{q}^{J}-x_{q}^{J}\right|+L_{f}^{K}\left|y_{q}^{K}-x_{q}^{K}\right|, \\
& \left|g_{q}^{l}\left(y_{q}^{R}, y_{q}^{I}, y_{q}^{J}, y_{q}^{K}\right)-g_{q}^{l}\left(x_{q}^{R}, x_{q}^{I}, x_{q}^{J}, x_{q}^{K}\right)\right| \\
& \quad \leq L_{g}^{R}\left|y_{q}^{R}-x_{q}^{R}\right|+L_{g}^{I}\left|y_{q}^{I}-x_{q}^{I}\right|+L_{g}^{J}\left|y_{q}^{J}-x_{q}^{J}\right|+L_{g}^{K}\left|y_{q}^{K}-x_{q}^{K}\right|,
\end{aligned}
$$

and $f_{q}^{l}(\mathbf{0})=g_{q}^{l}(\mathbf{0})=0$, where $q \in S, l \in T$.

$\left(H_{3}\right) \rho=\max _{p \in S}\left\{\frac{1}{c_{p}^{-}}\left(A_{p}+B_{p}\right)\right\}<1$, where for $p \in S$,

$$
\begin{aligned}
& A_{p}=\sum_{q=1}^{n}\left(a_{p q}^{R^{+}}+a_{p q}^{I^{+}}+a_{p q}^{J^{+}}+a_{p q}^{K^{+}}\right)\left(L_{f}^{R}+L_{f}^{I}+L_{f}^{J}+L_{f}^{K}\right), \\
& B_{p}=\sum_{q=1}^{n}\left(b_{p q}^{R^{+}}+b_{p q}^{I^{+}}+b_{p q}^{J^{+}}+b_{p q}^{K^{+}}\right)\left(L_{g}^{R}+L_{g}^{I}+L_{g}^{J}+L_{g}^{K}\right) .
\end{aligned}
$$

\section{The existence of weighted pseudo-almost periodic solutions}

In this section, we will study the existence and global exponential stability of weighted pseudo-almost periodic solutions of system (2).

Let

$$
\mathbb{B}=\left\{\varphi=\left(\varphi_{1}^{R}, \ldots, \varphi_{n}^{R}, \varphi_{1}^{I}, \ldots, \varphi_{n}^{I}, \varphi_{1}^{J}, \ldots, \varphi_{n}^{J}, \varphi_{1}^{K}, \ldots,\right.\right.
$$




$$
\left.\left.\varphi_{n}^{K}\right)^{T}:=\left(\varphi_{1}, \varphi_{2}, \ldots, \varphi_{n}\right)^{T} \in P A P\left(\mathbb{R}, \mathbb{R}^{4 n}, v\right)\right\}
$$

with the norm $\|\varphi\|_{\mathbb{B}}=\max _{p \in S}\left\{\max _{l \in T}\left\{\sup _{t \in \mathbb{R}}\left|\varphi_{p}^{l}(t)\right|\right\}\right\}$, then $\mathbb{B}$ is a Banach space.

Let

$$
\begin{aligned}
\varphi^{0}(t)= & \left(\left(\varphi^{0}\right)_{1}^{R}(t), \ldots,\left(\varphi^{0}\right)_{n}^{R}(t),\left(\varphi^{0}\right)_{1}^{I}(t), \ldots,\left(\varphi^{0}\right)_{n}^{I}(t),\right. \\
& \left.\left(\varphi^{0}\right)_{1}^{J}(t), \ldots,\left(\varphi^{0}\right)_{n}^{J}(t),\left(\varphi^{0}\right)_{1}^{K}(t), \ldots,\left(\varphi^{0}\right)_{n}^{K}(t)\right)^{T},
\end{aligned}
$$

where $\left(\varphi^{0}\right)_{p}^{l}(t)=\int_{-\infty}^{t} e^{-\int_{s}^{t} c_{p}(u) \mathrm{d} u} J_{p}^{l}(s) \mathrm{d} s, p \in S, l \in T$ and $\kappa$ is a constant satisfying $\kappa \geq$ $\left\|\varphi^{0}\right\|_{\mathbb{B}}$.

Lemma 4 Fix $v \in \mathbb{W}_{\infty}^{I n v}$. Suppose that assumptions $\left(H_{1}\right)$ and $\left(H_{2}\right)$ hold. For each $\varphi=$ $\left(\varphi_{1}^{R}, \ldots, \varphi_{n}^{R}, \varphi_{1}^{I}, \ldots, \varphi_{n}^{I}, \varphi_{1}^{J}, \ldots, \varphi_{n}^{J}, \varphi_{1}^{K}, \ldots, \varphi_{n}^{K}\right)^{T} \in \mathbb{B}$, define a nonlinear operator $\Phi$ as follows:

$$
\begin{aligned}
& \left(\varphi_{1}^{R}, \ldots, \varphi_{n}^{R}, \varphi_{1}^{I}, \ldots, \varphi_{n}^{I}, \varphi_{1}^{J}, \ldots, \varphi_{n}^{J}, \varphi_{1}^{K}, \ldots, \varphi_{n}^{K}\right)^{T} \\
& \quad \rightarrow\left(\left(x^{\varphi}\right)_{1}^{R}, \ldots,\left(x^{\varphi}\right)_{n}^{R},\left(x^{\varphi}\right)_{1}^{I}, \ldots,\left(x^{\varphi}\right)_{n}^{I},\left(x^{\varphi}\right)_{1}^{J}, \ldots,\left(x^{\varphi}\right)_{n}^{J},\left(x^{\varphi}\right)_{1}^{K}, \ldots,\left(x^{\varphi}\right)_{n}^{K}\right)^{T},
\end{aligned}
$$

where

$$
\left(x^{\varphi}\right)_{p}^{l}(t)=\int_{-\infty}^{t} e^{-\int_{s}^{t} c_{p}(u) \mathrm{d} u} \Omega_{p}^{l}(s) \mathrm{d} s, \quad \Omega_{p}^{l}(t)=F_{p}^{l}(t, \varphi(t))+J_{p}^{l}(t), \quad p \in S, l \in T,
$$

then $\Phi$ maps $\mathbb{B}$ into itself.

Proof Let $\varphi \in \mathbb{B}$. By $\left(H_{2}\right)$ and Lemma 3, we have $f_{q}^{l}[t, \varphi] \in P A P(\mathbb{R}, \mathbb{R}, v)$ and by $\left(H_{1}\right)$ and Lemma 3, we have $g_{q}^{l}[t, \tau, \varphi] \in P A P(\mathbb{R}, \mathbb{R}, v)$. Hence, from Lemma 1 , we obtain $\Omega_{p}^{l} \in$ $\operatorname{PAP}(\mathbb{R}, \mathbb{R}, v)$ for all $p \in S, l \in T$. Consequently, $\Omega_{p}^{l}$ can be written as $\Omega_{p}^{l}=\Omega_{p 1}^{l}+\Omega_{p 2}^{l}$, where $\Omega_{p 1}^{l} \in A P(\mathbb{R}, \mathbb{R}), \Omega_{p 2}^{l} \in P A P_{0}(\mathbb{R}, \mathbb{R}, v)$. Hence,

$$
\begin{aligned}
\left(x^{\varphi}\right)_{p}^{l}(t) & =\int_{-\infty}^{t} e^{-\int_{s}^{t} c_{p}(u) \mathrm{d} u} \Omega_{p 1}^{l}(s) \mathrm{d} s+\int_{-\infty}^{t} e^{-\int_{s}^{t} c_{p}(u) \mathrm{d} u} \Omega_{p 2}^{l}(s) \mathrm{d} s \\
& =: \Theta_{p 1}^{l}(t)+\Theta_{p 2}^{l}(t), \quad p \in S, l \in T .
\end{aligned}
$$

First, we will prove that $\Theta_{p 1}^{l} \in A P(\mathbb{R}, \mathbb{R})$ for all $p \in S, l \in T$. For every $\epsilon>0$, since $\Omega_{p 1}^{l}, c_{p} \epsilon$ $A P(\mathbb{R}, \mathbb{R})$, it is possible to find a real number $l=l(\epsilon)>0$, for each interval with length $l(\epsilon)$, there exists a number $\tau=\tau(\epsilon)$ in this interval such that $\left|\Omega_{p 1}^{l}(t+\tau)-\Omega_{p 1}^{l}(t)\right|<\epsilon$ and $\left|c_{p}(t+\tau)-c_{p}(t)\right|<\epsilon$, then

$$
\begin{aligned}
& \left|\Theta_{p 1}^{l}(t+\tau)-\Theta_{p 1}^{l}(t)\right| \\
& \quad=\left|\int_{-\infty}^{t+\tau} e^{-\int_{s}^{t+\tau} c_{p}(u) \mathrm{d} u} \Omega_{p 1}^{l}(s) \mathrm{d} s-\int_{-\infty}^{t} e^{-\int_{s}^{t} c_{p}(u) \mathrm{d} u} \Omega_{p 1}^{l}(s) \mathrm{d} s\right| \\
& \quad=\left|\int_{-\infty}^{t} e^{-\int_{s+\tau}^{t+\tau} c_{p}(u) \mathrm{d} u} \Omega_{p 1}^{l}(s+\tau) \mathrm{d} s-\int_{-\infty}^{t} e^{-\int_{s}^{t} c_{p}(u) \mathrm{d} u} \Omega_{p 1}^{l}(s) \mathrm{d} s\right| \\
& \quad=\mid \int_{-\infty}^{t} e^{-\int_{s+\tau}^{t+\tau} c_{p}(u) \mathrm{d} u} \Omega_{p 1}^{l}(s+\tau) \mathrm{d} s-\int_{-\infty}^{t} e^{-\int_{s}^{t} c_{p}(u) \mathrm{d} u} \Omega_{p 1}^{l}(s+\tau) \mathrm{d} s
\end{aligned}
$$




$$
\begin{aligned}
& +\int_{-\infty}^{t} e^{-\int_{s}^{t} c_{p}(u) \mathrm{d} u} \Omega_{p 1}^{l}(s+\tau) \mathrm{d} s-\int_{-\infty}^{t} e^{-\int_{s}^{t} c_{p}(u) \mathrm{d} u} \Omega_{p 1}^{l}(s) \mathrm{d} s \mid \\
\leq & \int_{-\infty}^{t}\left|e^{-\int_{s+\tau}^{t+\tau} c_{p}(u) \mathrm{d} u}-e^{-\int_{s}^{t} c_{p}(u) \mathrm{d} u}\right|\left|\Omega_{p 1}^{l}(s+\tau)\right| \mathrm{d} s \\
& +\int_{-\infty}^{t} e^{-\int_{s}^{t} c_{p}(u) \mathrm{d} u}\left|\Omega_{p 1}^{l}(s+\tau)-\Omega_{p 1}^{l}(s)\right| \mathrm{d} s .
\end{aligned}
$$

By $\left(e^{-\int_{s}^{t} c_{p}(u) \mathrm{d} u}\right)_{t}^{\prime}=-c_{p}(t) e^{-\int_{s}^{t} c_{p}(u) \mathrm{d} u}$, we have

$$
\begin{aligned}
& \left|e^{-\int_{s+\tau}^{t+\tau} c_{p}(u) \mathrm{d} u}-e^{-\int_{s}^{t} c_{p}(u) \mathrm{d} u}\right| \\
& =\left|-\left(e^{-\int_{\theta}^{t} c_{p}(u) \mathrm{d} u} e^{-\int_{s+\tau}^{\theta+\tau} c_{p}(u) \mathrm{d} u}\right)\right|_{\theta=t}^{s} \mid \\
& =\mid-\left[\int_{t}^{s} e^{-\int_{\theta}^{t} c_{p}(u) \mathrm{d} u}\left(e^{-\int_{s+\tau}^{\theta+\tau} c_{p}(u) \mathrm{d} u}\right)_{\theta}^{\prime} \mathrm{d} \theta\right. \\
& \left.+\int_{t}^{s}\left(e^{-\int_{\theta}^{t} c_{p}(u) \mathrm{d} u}\right)_{\theta}^{\prime} e^{-\int_{s+\tau}^{\theta+\tau} c_{p}(u) \mathrm{d} u} \mathrm{~d} \theta\right] \\
& =\left|\int_{t}^{s} e^{-\int_{\theta}^{t} c_{p}(u) \mathrm{d} u}\left(c_{p}(\theta+\tau)-c_{p}(\theta)\right) e^{-\int_{s+\tau}^{\theta+\tau} c_{p}(u) \mathrm{d} u} \mathrm{~d} \theta\right| \\
& \leq \int_{t}^{s} e^{-\int_{\theta}^{t} c_{p}(u) \mathrm{d} u}\left|c_{p}(\theta+\tau)-c_{p}(\theta)\right| \mathrm{d} \theta \leq \frac{1}{c_{p}^{-}} e^{-\int_{s}^{t} c_{p}(u) \mathrm{d} u} \epsilon .
\end{aligned}
$$

Since $\Omega_{p 1}^{l} \in A P(\mathbb{R}, \mathbb{R})$, it is a uniformly continuous and bounded function. Denote $G(t):=$ $\int_{-\infty}^{t}\left|\Omega_{p 1}^{l}(s)\right| \mathrm{d} s$ and substitute (4) into (3), we have

$$
\left|\Theta_{p 1}^{l}(t+\tau)-\Theta_{p 1}^{l}(t)\right| \leq \frac{\epsilon}{\left(c_{p}^{-}\right)^{2}}\left(c_{p}^{-}+\|G\|_{\mathbb{B}}\right),
$$

which implies that $\Theta_{p 1}^{l} \in A P(\mathbb{R}, \mathbb{R}), p \in S, l \in T$.

Next, for $p \in S, l \in T$, set

$$
\Lambda_{p}^{l}=\frac{1}{v\left(Q_{r}\right)} \int_{Q_{r}}\left|\int_{-\infty}^{t} e^{-\int_{s}^{t} c_{p}(u) \mathrm{d} u} \Omega_{p 2}^{l}(s) \mathrm{d} s\right| v(t) \mathrm{d} t
$$

To prove that $\Theta_{p 2}^{l} \in P A P_{0}(\mathbb{R}, \mathbb{R}, v)$, we only need to show that $\lim _{r \rightarrow \infty} \Lambda_{p}^{l}=0, p \in S, l \in T$. By a similar argument as that in the proof of Lemma 3.4 in [47], one can see that $\Theta_{p 2}^{l} \in$ $P A P_{0}(\mathbb{R}, \mathbb{R}, \nu), p \in S, l \in T$. Therefore, we have $\left(x^{\varphi}\right)_{p}^{l} \in P A P(\mathbb{R}, \mathbb{R}, \nu)$, that is, $\Phi$ maps $\mathbb{B}$ into itself. This completes the proof.

Remark 2 It is easy to check that, for $p \in S, l \in T$,

$$
\left(x^{\varphi}\right)_{p}^{l}(t)=\int_{-\infty}^{t} e^{-\int_{s}^{t} c_{p}(u) \mathrm{d} u} \Omega_{p}^{l}(s) \mathrm{d} s
$$

satisfy the following equations:

$$
\left(x_{p}^{l}\right)^{\prime}(t)=-c_{p}(t) x_{p}^{l}(t)+\Omega_{p}^{l}(t), \quad p \in S, l \in T .
$$


Theorem 1 Assume that $\left(H_{1}\right)-\left(H_{3}\right)$ hold, then system (2) has a unique weighted pseudoalmost periodic solution in $\mathbb{B}^{*}=\left\{\varphi \mid \varphi \in \mathbb{B},\left\|\varphi-\varphi^{0}\right\|_{\mathbb{B}} \leq \frac{\rho \kappa}{1-\rho}\right\}$.

Proof For any $\varphi \in \mathbb{B}$, by Lemma 4 , $\Phi$ maps $\mathbb{B}$ into itself. Obviously,

$$
\begin{aligned}
\left\|\varphi^{0}\right\|_{\mathbb{B}} & =\max _{1 \leq p \leq n}\left\{\sup _{t \in \mathbb{R}} \max _{l \in T}\left|\int_{-\infty}^{t} e^{-\int_{s}^{t} c_{p}(u) \mathrm{d} u} J_{p}^{l}(s) \mathrm{d} s\right|\right\} \\
& \leq \max _{1 \leq p \leq n}\left\{\max _{l \in T}\left\{\frac{J_{p}^{l^{+}}}{c_{p}^{-}}\right\}\right\}=\kappa .
\end{aligned}
$$

Hence, for all $\varphi \in \mathbb{B}^{*}=\left\{\varphi \mid \varphi \in \mathbb{B},\left\|\varphi-\varphi^{0}\right\|_{\mathbb{B}} \leq \frac{\rho \kappa}{1-\rho}\right\}$, we have

$$
\|\varphi\|_{\mathbb{B}} \leq\left\|\varphi-\varphi^{0}\right\|_{\mathbb{B}}+\left\|\varphi^{0}\right\|_{\mathbb{B}} \leq \frac{\rho \kappa}{1-\rho}+\kappa=\frac{\kappa}{1-\rho} .
$$

Next, we show that $\Phi$ maps $\mathbb{B}^{*}$ into itself. In fact, for any $\varphi \in \mathbb{B}^{*}$, by $\left(H_{2}\right)$, we have

$$
\begin{aligned}
& \sup _{t \in \mathbb{R}}\left|(\Phi \varphi)_{p}^{R}(t)-\left(\varphi^{0}\right)_{p}^{R}(t)\right|
\end{aligned}
$$

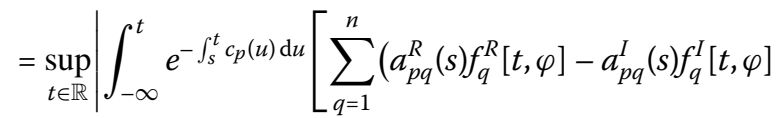

$$
\begin{aligned}
& \left.-a_{p q}^{J}(s) f_{q}^{J}[t, \varphi]-a_{p q}^{K}(s) f_{q}^{K}[t, \varphi]\right)+\sum_{q=1}^{n}\left(b_{p q}^{R}(s) g_{q}^{R}[t, \tau, \varphi]\right. \\
& \left.\left.-b_{p q}^{I}(s) g_{q}^{I}[t, \tau, \varphi]-b_{p q}^{J}(s) g_{q}^{J}[t, \tau, \varphi]-b_{p q}^{K}(s) g_{q}^{K}[t, \tau, \varphi]\right)\right] \mathrm{d} s \mid
\end{aligned}
$$

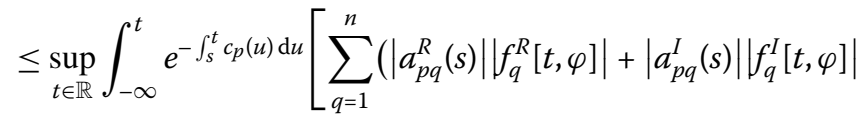

$$
\begin{aligned}
& \left.+\left|a_{p q}^{J}(s)\right|\left|f_{q}^{J}[t, \varphi]\right|+\left|a_{p q}^{K}(s)\right|\left|f_{q}^{K}[t, \varphi]\right|\right)+\sum_{q=1}^{n}\left(\left|b_{p q}^{R}(s)\right|\left|g_{q}^{R}[t, \tau, \varphi]\right|\right. \\
& \left.\left.+\left|b_{p q}^{I}(s)\right|\left|g_{q}^{I}[t, \tau, \varphi]\right|+\left|b_{p q}^{J}(s)\right|\left|g_{q}^{J}[t, \tau, \varphi]\right|+\left|b_{p q}^{K}(s)\right|\left|g_{q}^{K}[t, \tau, \varphi]\right|\right)\right] \mathrm{d} s
\end{aligned}
$$

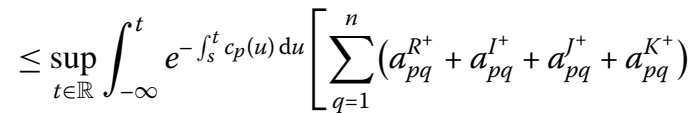

$$
\begin{aligned}
& \times\left(L_{f}^{R}+L_{f}^{I}+L_{f}^{J}+L_{f}^{K}\right)\|\varphi\|_{\mathbb{B}}+\sum_{q=1}^{n}\left(b_{p q}^{R^{+}}+b_{p q}^{I^{+}}+b_{p q}^{J^{+}}+b_{p q}^{K^{+}}\right) \\
& \left.\times\left(L_{g}^{R}+L_{g}^{I}+L_{g}^{J}+L_{g}^{K}\right)\|\varphi\|_{\mathbb{B}}\right] \mathrm{d} s \\
& \leq \frac{1}{c_{p}^{-}}\left(A_{p}+B_{p}\right)\|\varphi\|_{\mathbb{B}}, \quad p \in S \text {. }
\end{aligned}
$$


Similarly, we can obtain

$$
\sup _{t \in \mathbb{R}}\left|(\Phi \varphi)_{p}^{l}(t)-\left(\varphi^{0}\right)_{p}^{l}(t)\right| \leq \frac{1}{c_{p}^{-}}\left(A_{p}+B_{p}\right)\|\varphi\|_{\mathbb{B}}, \quad p \in S, l=I, J, K .
$$

It follows from (5) and (6) that

$$
\left\|\Phi \varphi-\varphi^{0}\right\|_{\mathbb{B}} \leq \rho\|\varphi\|_{\mathbb{B}} \leq \frac{\rho \kappa}{1-\rho},
$$

which implies that $\Phi \varphi \in \mathbb{B}^{*}$. So, the mapping $\Phi$ is a self mapping from $\mathbb{B}^{*}$ to $\mathbb{B}^{*}$. Finally, we prove that $\Phi$ is a contraction mapping. In fact, in view of $\left(H_{2}\right)$, for any $\varphi, \psi \in \mathbb{B}$, we have

$$
\begin{aligned}
& \sup _{t \in \mathbb{R}}\left|(\Phi \varphi)_{p}^{R}(t)-(\Phi \psi)_{p}^{R}(t)\right| \\
& =\sup _{t \in \mathbb{R}} \mid \int_{-\infty}^{t} e^{-\int_{s}^{t} c_{p}(u) \mathrm{d} u}\left[\sum _ { q = 1 } ^ { n } \left(a_{p q}^{R}(s)\left(f_{q}^{R}\{t, \varphi\}-f_{q}^{R}\{t, \psi\}\right)\right.\right. \\
& -a_{p q}^{I}(s)\left(f_{q}^{I}\{t, \varphi\}-f_{q}^{I}\{t, \psi\}\right)-a_{p q}^{J}(s)\left(f_{q}^{J}\{t, \varphi\}-f_{q}^{J}\{t, \psi\}\right) \\
& \left.-a_{p q}^{K}(s)\left(f_{q}^{K}\{t, \varphi\}-f_{q}^{K}\{t, \psi\}\right)\right)+\sum_{q=1}^{n}\left(b _ { p q } ^ { R } ( s ) \left(g_{q}^{R}\{t, \tau, \varphi\}\right.\right. \\
& \left.-g_{q}^{R}\{t, \tau, \psi\}\right)-b_{p q}^{I}(s)\left(g_{q}^{I}\{t, \tau, \varphi\}-g_{q}^{I}\{t, \tau, \psi\}\right) \\
& -b_{p q}^{J}(s)\left(g_{q}^{J}\{t, \tau, \varphi\}-g_{q}^{J}\{t, \tau, \psi\}\right)-b_{p q}^{K}(s)\left(g_{q}^{K}\{t, \tau, \varphi\}\right. \\
& \left.\left.\left.-g_{q}^{K}\{t, \tau, \psi\}\right)\right)\right] \mathrm{d} s
\end{aligned}
$$

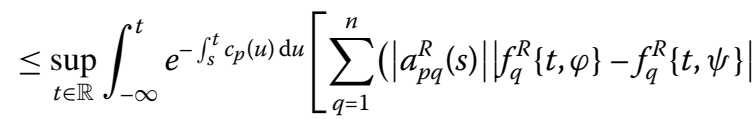

$$
\begin{aligned}
& +\left|a_{p q}^{I}(s)\right|\left|f_{q}^{I}\{t, \varphi\}-f_{q}^{I}\{t, \psi\}\right|+\left|a_{p q}^{J}(s)\right|\left|f_{q}^{J}\{t, \varphi\}-f_{q}^{J}\{t, \psi\}\right| \\
& \left.+\left|a_{p q}^{K}(s)\right|\left|f_{q}^{K}\{t, \varphi\}-f_{q}^{K}\{t, \psi\}\right|\right)+\sum_{q=1}^{n}\left(\left|b_{p q}^{R}(s)\right| \mid g_{q}^{R}\{t, \tau, \varphi\}\right. \\
& -g_{q}^{R}\{t, \tau, \psi\}|+| b_{p q}^{I}(s)|| g_{q}^{I}\{t, \tau, \varphi\}-g_{q}^{I}\{t, \tau, \psi\} \mid \\
& +\left|b_{p q}^{J}(s)\right|\left|g_{q}^{J}\{t, \tau, \varphi\}-g_{q}^{J}\{t, \tau, \psi\}\right|+\left|b_{p q}^{K}(s)\right| \mid g_{q}^{K}\{t, \tau, \varphi\} \\
& \left.\left.-g_{q}^{K}\{t, \tau, \psi\} \mid\right)\right] \mathrm{d} s
\end{aligned}
$$

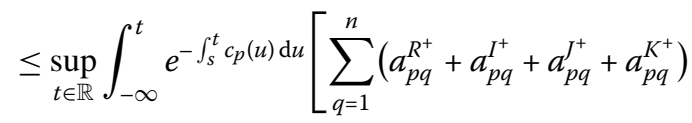

$$
\begin{aligned}
& \times\left(L_{f}^{R}+L_{f}^{I}+L_{f}^{J}+L_{f}^{K}\right)\|\varphi-\psi\|_{\mathbb{B}}+\sum_{q=1}^{n}\left(b_{p q}^{R^{+}}+b_{p q}^{I^{+}}\right. \\
& \left.\left.+b_{p q}^{J^{+}}+b_{p q}^{K^{+}}\right)\left(L_{g}^{R}+L_{g}^{I}+L_{g}^{J}+L_{g}^{K}\right)\|\varphi-\psi\|_{\mathbb{B}}\right] \mathrm{d} s \\
& \leq \frac{1}{c_{p}^{-}}\left(A_{p}+B_{p}\right)\|\varphi-\psi\|_{\mathbb{B}}, \quad p \in S .
\end{aligned}
$$


Similarly, we can get

$$
\sup _{t \in \mathbb{R}}\left|(\Phi \varphi)_{p}^{R}(t)-(\Phi \psi)_{p}^{R}(t)\right| \leq \frac{1}{c_{p}^{-}}\left(A_{p}+B_{p}\right)\|\varphi-\psi\|_{\mathbb{B}}, \quad p \in S, l=I, J, K .
$$

From (7) and (8), we obtain

$$
\|\Phi \varphi-\Phi \psi\|_{\mathbb{B}} \leq \rho\|\varphi-\psi\|_{\mathbb{B}} .
$$

Since $\left(H_{3}\right), \Phi$ is a contraction mapping. Hence, $\Phi$ has a fixed point in $\mathbb{B}^{*}$. That is, system (2) has a unique weighted pseudo-almost periodic solution in $\mathbb{B}^{*}$. This completes the proof.

\section{Global exponential synchronization}

In this section, we consider system (1) as the drive system and design a response system as

$$
\begin{aligned}
y_{p}^{\prime}(t)= & -c_{p}(t) y_{p}(t)+\sum_{q=1}^{n} a_{p q}(t) f_{q}\left(y_{q}(t)\right) \\
& +\sum_{q=1}^{n} b_{p q}(t) g_{q}\left(y_{q}\left(t-\tau_{p q}(t)\right)\right)+J_{p}(t)+u_{p}(t),
\end{aligned}
$$

where $p \in S, u_{p}(t)$ is a controlled input.

Let signals $e_{p}(t)=y_{p}(t)-x_{p}(t)$, then we can obtain the following error system:

$$
\begin{aligned}
e_{p}^{\prime}(t)= & -c_{p}(t) e_{p}(t)+\sum_{q=1}^{n} a_{p q}(t)\left(f_{q}\left(y_{q}(t)\right)-f_{q}\left(x_{q}(t)\right)\right)+\sum_{q=1}^{n} b_{p q}(t) \\
& \times\left(g_{q}\left(y_{q}\left(t-\tau_{p q}(t)\right)\right)-g_{q}\left(x_{q}\left(t-\tau_{p q}(t)\right)\right)\right)+u_{p}(t), \quad p \in S .
\end{aligned}
$$

In order to realize the weighted pseudo-almost periodic synchronization of the driveresponse system, we design the following state-feedback controller:

$$
u_{p}(t)=-d_{p}(t) e_{p}(t)+\sum_{q=1}^{n} p_{p q}(t) h_{q}\left(e_{q}(t)\right)+\sum_{q=1}^{n} q_{p q}(t) \bar{h}_{q}\left(e_{q}\left(t-\sigma_{p q}(t)\right)\right), \quad p \in S .
$$

The initial condition of (9) is

$$
y_{p}(s)=\psi_{p}(s), \quad s \in[-\xi, 0], p \in S,
$$

where $\xi=\max \{\tau, \sigma\}, \sigma=\max _{p, q \in S}\left\{\sup _{t \in \mathbb{R}} \sigma_{p q}(t)\right\}, \psi_{p}(s)=\psi_{p}^{R}(s)+i \psi_{p}^{I}(s)+j \psi_{p}^{J}(s)+k \psi_{p}^{K}(s)$ is a continuous function.

System (10) can be decomposed into the following real-valued system:

$$
\begin{aligned}
\left(e_{p}^{R}\right)^{\prime}(t)= & -\left(c_{p}(t)+d_{p}(t)\right) e_{p}^{R}(t)+\sum_{q=1}^{n}\left(a_{p q}^{R}(t)\left(f_{q}^{R}\{t, y\}-f_{q}^{R}\{t, x\}\right)\right. \\
& -a_{p q}^{I}(t)\left(f_{q}^{I}\{t, y\}-f_{q}^{I}\{t, x\}\right)-a_{p q}^{J}(t)\left(f_{q}^{I}\{t, y\}-f_{q}^{J}\{t, x\}\right)
\end{aligned}
$$




$$
\begin{aligned}
& \left.-a_{p q}^{K}(t)\left(f_{q}^{K}\{t, y\}-f_{q}^{K}\{t, x\}\right)\right)+\sum_{q=1}^{n}\left(b _ { p q } ^ { R } ( t ) \left(g_{q}^{R}\{t, y\}\right.\right. \\
& \left.-g_{q}^{R}\{t, x\}\right)-b_{p q}^{I}(t)\left(g_{q}^{I}\{t, y\}-g_{q}^{I}\{t, x\}\right)-b_{p q}^{J}(t)\left(g_{q}^{J}\{t, y\}\right. \\
& \left.\left.-g_{q}^{J}\{t, x\}\right)-b_{p q}^{K}(t)\left(g_{q}^{K}\{t, y\}-g_{q}^{K}\{t, x\}\right)\right) \\
& +\sum_{q=1}^{n}\left(p_{p q}^{R}(t) h_{q}^{R}\{t, e\}-p_{p q}^{I}(t) h_{q}^{I}\{t, e\}-p_{p q}^{J}(t) h_{q}^{J}\{t, e\}\right. \\
& \left.-p_{p q}^{K}(t) h_{q}^{K}\{t, e\}\right)+\sum_{q=1}^{n}\left(q_{p q}^{R}(t) \bar{h}_{q}^{R}\{t, \sigma, e\}-q_{p q}^{I}(t) \bar{h}_{q}^{I}\{t, \sigma, e\}\right. \\
& \left.-q_{p q}^{J}(t) \bar{h}_{q}^{J}\{t, \sigma, e\}-q_{p q}^{K}(t) \bar{h}_{q}^{K}\{t, \sigma, e\}\right), \\
& \left(e_{p}^{I}\right)^{\prime}(t)=-\left(c_{p}(t)+d_{p}(t)\right) e_{p}^{I}(t)+\sum_{q=1}^{n}\left(a_{p q}^{R}(t)\left(f_{q}^{I}\{t, y\}-f_{q}^{I}\{t, x\}\right)\right. \\
& +a_{p q}^{I}(t)\left(f_{q}^{R}\{t, y\}-f_{q}^{R}\{t, x\}\right)+a_{p q}^{J}(t)\left(f_{q}^{K}\{t, y\}-f_{q}^{K}\{t, x\}\right) \\
& \left.-a_{p q}^{K}(t)\left(f_{q}^{J}\{t, y\}-f_{q}^{J}\{t, x\}\right)\right)+\sum_{q=1}^{n}\left(b _ { p q } ^ { R } ( t ) \left(g_{q}^{I}\{t, y\}\right.\right. \\
& \left.-g_{q}^{I}\{t, x\}\right)+b_{p q}^{I}(t)\left(g_{q}^{R}\{t, y\}-g_{q}^{R}\{t, x\}\right)+b_{p q}^{J}(t)\left(g_{q}^{K}\{t, y\}\right. \\
& \left.\left.-g_{q}^{K}\{t, x\}\right)-b_{p q}^{K}(t)\left(g_{q}^{J}\{t, y\}-g_{q}^{J}\{t, x\}\right)\right) \\
& +\sum_{q=1}^{n}\left(p_{p q}^{R}(t) h_{q}^{I}\{t, e\}+p_{p q}^{I}(t) h_{q}^{R}\{t, e\}+p_{p q}^{J}(t) h_{q}^{K}\{t, e\}\right. \\
& \left.-p_{p q}^{K}(t) h_{q}^{J}\{t, e\}\right)+\sum_{q=1}^{n}\left(q_{p q}^{R}(t) \bar{h}_{q}^{I}\{t, \sigma, e\}+q_{p q}^{I}(t) \bar{h}_{q}^{R}\{t, \sigma, e\}\right. \\
& \left.+q_{p q}^{J}(t) \bar{h}_{q}^{K}\{t, \sigma, e\}-q_{p q}^{K}(t) \bar{h}_{q}^{J}\{t, \sigma, e\}\right), \\
& \left(e_{p}^{J}\right)^{\prime}(t)=-\left(c_{p}(t)+d_{p}(t)\right) e_{p}^{J}(t)+\sum_{q=1}^{n}\left(a_{p q}^{R}(t)\left(f_{q}^{J}\{t, y\}-f_{q}^{J}\{t, x\}\right)\right. \\
& +a_{p q}^{J}(t)\left(f_{q}^{R}\{t, y\}-f_{q}^{R}\{t, x\}\right)-a_{p q}^{I}(t)\left(f_{q}^{K}\{t, y\}-f_{q}^{K}\{t, x\}\right) \\
& \left.+a_{p q}^{K}(t)\left(f_{q}^{I}\{t, y\}-f_{q}^{I}\{t, x\}\right)\right)+\sum_{q=1}^{n}\left(b _ { p q } ^ { R } ( t ) \left(g_{q}^{I}\{t, y\}\right.\right. \\
& \left.-g_{q}^{J}\{t, x\}\right)+b_{p q}^{J}(t)\left(g_{q}^{R}\{t, y\}-g_{q}^{R}\{t, x\}\right)-b_{p q}^{I}(t)\left(g_{q}^{K}\{t, y\}\right. \\
& \left.\left.-g_{q}^{K}\{t, x\}\right)+b_{p q}^{K}(t)\left(g_{q}^{I}\{t, y\}-g_{q}^{I}\{t, x\}\right)\right) \\
& +\sum_{q=1}^{n}\left(p_{p q}^{R}(t) h_{q}^{J}\{t, e\}+p_{p q}^{J}(t) h_{q}^{R}\{t, e\}-p_{p q}^{I}(t) h_{q}^{K}\{t, e\}\right. \\
& \left.+p_{p q}^{K}(t) h_{q}^{I}\{t, e\}\right)+\sum_{q=1}^{n}\left(q_{p q}^{R}(t) \bar{h}_{q}^{J}\{t, \sigma, e\}+q_{p q}^{J}(t) \bar{h}_{q}^{R}\{t, \sigma, e\}\right. \\
& \left.-q_{p q}^{I}(t) \bar{h}_{q}^{K}\{t, \sigma, e\}+q_{p q}^{K}(t) \bar{h}_{q}^{I}\{t, \sigma, e\}\right),
\end{aligned}
$$




$$
\begin{aligned}
\left(e_{p}^{K}\right)^{\prime}(t)= & -\left(c_{p}(t)+d_{p}(t)\right) e_{p}^{K}(t)+\sum_{q=1}^{n}\left(a_{p q}^{R}(t)\left(f_{q}^{K}\{t, y\}-f_{q}^{K}\{t, x\}\right)\right. \\
& +a_{p q}^{K}(t)\left(f_{q}^{R}\{t, y\}-f_{q}^{R}\{t, x\}\right)+a_{p q}^{I}(t)\left(f_{q}^{J}\{t, y\}-f_{q}^{J}\{t, x\}\right) \\
& \left.-a_{p q}^{J}(t)\left(f_{q}^{I}\{t, y\}-f_{q}^{I}\{t, x\}\right)\right)+\sum_{q=1}^{n}\left(b _ { p q } ^ { R } ( t ) \left(g_{q}^{K}\{t, y\}\right.\right. \\
& \left.-g_{q}^{K}\{t, x\}\right)+b_{p q}^{K}(t)\left(g_{q}^{R}\{t, y\}-g_{q}^{R}\{t, x\}\right)+b_{p q}^{I}(t)\left(g_{q}^{J}\{t, y\}\right. \\
& \left.\left.-g_{q}^{J}\{t, x\}\right)-b_{p q}^{J}(t)\left(g_{q}^{I}\{t, y\}-g_{q}^{I}\{t, x\}\right)\right) \\
& +\sum_{q=1}^{n}\left(p_{p q}^{R}(t) h_{q}^{K}\{t, e\}+p_{p q}^{K}(t) h_{q}^{R}\{t, e\}+p_{p q}^{I}(t) h_{q}^{J}\{t, e\}\right. \\
& \left.-p_{p q}^{J}(t) h_{q}^{I}\{t, e\}\right)+\sum_{q=1}^{n}\left(q_{p q}^{R}(t) \bar{h}_{q}^{K}\{t, \sigma, e\}+q_{p q}^{K}(t) \bar{h}_{q}^{R}\{t, \sigma, e\}\right. \\
& \left.+q_{p q}^{I}(t) \bar{h}_{q}^{I}\{t, \sigma, e\}-q_{p q}^{J}(t) \bar{h}_{q}^{I}\{t, \sigma, e\}\right),
\end{aligned}
$$

where $h_{q}^{l}\{t, e\} \triangleq h_{q}^{l}\left(e_{q}^{R}(t), e_{q}^{I}(t), e_{q}^{J}(t), e_{q}^{K}(t)\right), \bar{h}_{q}^{l}\{t, \sigma, e\} \triangleq \bar{h}_{q}^{l}\left(e_{q}^{R}\left(t-\sigma_{p q}(t)\right), e_{q}^{I}\left(t-\sigma_{p q}(t)\right), e_{q}^{J}(t-\right.$ $\left.\left.\sigma_{p q}(t)\right), e_{q}^{K}\left(t-\sigma_{p q}(t)\right)\right), p, q \in S, l \in T$.

Definition 4 Systems (9) and (1) are globally exponentially synchronized, if there exist positive constants $M$ and $\lambda$ such that

$$
\|y(t)-x(t)\| \leq M\|\psi-\varphi\|_{0} e^{-\lambda t}, \quad t \geq 0
$$

where $x=\left(x_{1}^{R}, \ldots, x_{n}^{R}, x_{1}^{I}, \ldots, x_{n}^{I}, x_{1}^{J}, \ldots, x_{n}^{J}, x_{1}^{K}, \ldots, x_{n}^{K}\right)$ and $y=\left(y_{1}^{R}, \ldots, y_{n}^{R}, y_{1}^{I}, \ldots, y_{n}^{I}, y_{1}^{J}, \ldots\right.$, $\left.y_{n}^{J}, y_{1}^{K}, \ldots, y_{n}^{K}\right)$ are solutions of the corresponding real-valued systems of (1) and (9) with initial values $\varphi=\left(\varphi_{1}^{R}, \ldots, \varphi_{n}^{R}, \varphi_{1}^{I}, \ldots, \varphi_{n}^{I}, \varphi_{1}^{I}, \ldots, \varphi_{n}^{J}, \varphi_{1}^{K}, \ldots, \varphi_{n}^{K}\right)$ and $\psi=\left(\psi_{1}^{R}, \ldots, \psi_{n}^{R}, \psi_{1}^{I}, \ldots\right.$, $\left.\psi_{n}^{I}, \psi_{1}^{J}, \ldots, \psi_{n}^{J}, \psi_{1}^{K}, \ldots, \psi_{n}^{K}\right)$, respectively,

$$
\|y(t)-x(t)\|=\max _{p \in S, l \in T}\left\{\left|y_{p}^{l}(t)\right|-\left|x_{p}^{l}(t)\right|\right\}, \quad\|\psi-\varphi\|_{0}=\max _{p \in S, l \in T}\left\{\sup _{t \in \mathbb{R}}\left|\psi_{p}^{l}(t)\right|-\left|\varphi_{p}^{l}(t)\right|\right\} .
$$

Theorem 2 Let $\left(H_{1}\right)-\left(H_{3}\right)$ hold. Suppose further that

$\left(H_{4}\right)$ For $p, q \in S, d_{p} \in A P\left(\mathbb{R}, \mathbb{R}^{+}\right), p_{p q}, q_{p q} \in P A P(\mathbb{R}, \mathbb{H}), \sigma_{p q} \in A P\left(\mathbb{R}, \mathbb{R}^{+}\right)$.

$\left(H_{5}\right)$ Functions $h_{q}^{l}, \bar{h}_{q}^{l} \in C\left(\mathbb{R}^{4}, \mathbb{R}\right)$, for any $x_{q}^{l}, y_{q}^{l} \in \mathbb{R}$, there exist positive constants $L_{h}^{l}, L_{\bar{h}}^{l}$ such that, for $q \in S, l \in T$,

$$
\begin{aligned}
& \left|h_{q}^{l}\left(y_{q}^{R}, y_{q}^{I}, y_{q}^{J}, y_{q}^{K}\right)-h_{q}^{l}\left(x_{q}^{R}, x_{q}^{I}, x_{q}^{J}, x_{q}^{K}\right)\right| \\
& \quad \leq L_{h}^{R}\left|y_{q}^{R}-x_{q}^{R}\right|+L_{h}^{I}\left|y_{q}^{I}-x_{q}^{I}\right|+L_{h}^{J}\left|y_{q}^{J}-x_{q}^{J}\right|+L_{h}^{K}\left|y_{q}^{K}-x_{q}^{K}\right|, \\
& \left|\bar{h}_{q}^{l}\left(y_{q}^{R}, y_{q}^{I}, y_{q}^{J}, y_{q}^{K}\right)-\bar{h}_{q}^{l}\left(x_{q}^{R}, x_{q}^{I}, x_{q}^{J}, x_{q}^{K}\right)\right| \\
& \quad \leq L_{\bar{h}}^{R}\left|y_{q}^{R}-x_{q}^{R}\right|+L_{\bar{h}}^{I}\left|y_{q}^{I}-x_{q}^{I}\right|+L_{\bar{h}}^{J}\left|y_{q}^{J}-x_{q}^{J}\right|+L_{\bar{h}}^{K}\left|y_{q}^{K}-x_{q}^{K}\right| .
\end{aligned}
$$

$\left(H_{6}\right)$ There exists a positive constant $\lambda$ such that

$$
\lambda-c_{p}^{-}-d_{p}^{-}+A_{p}+\frac{1}{\alpha} B_{p} e^{\lambda \tau}+P_{p}+\frac{1}{\beta} Q_{p} e^{\lambda \sigma}<0, \quad p \in S,
$$


where

$$
\begin{aligned}
& P_{p}=\sum_{q=1}^{n}\left(p_{p q}^{R^{+}}+p_{p q}^{I^{+}}+p_{p q}^{J^{+}}+p_{p q}^{K^{+}}\right)\left(L_{h}^{R}+L_{h}^{I}+L_{h}^{J}+L_{h}^{K}\right), \\
& Q_{p}=\sum_{q=1}^{n}\left(q_{p q}^{R^{+}}+q_{p q}^{I^{+}}+q_{p q}^{J^{+}}+q_{p q}^{K^{+}}\right)\left(L_{\bar{h}}^{R}+L_{\bar{h}}^{I}+L_{\bar{h}}^{J}+L_{\bar{h}}^{K}\right) .
\end{aligned}
$$

Then (1) has a unique weighted pseudo-almost periodic solution. Moreover, (1) and (9) are globally exponentially synchronized.

Proof By (10), for any $t>0, l \in T$, we have

$$
\begin{aligned}
& D^{+}\left|e_{p}^{l}(t)\right| \\
& \leq-\left(c_{p}^{-}+d_{p}^{-}\right)\left|e_{p}^{l}(t)\right|+\sum_{q=1}^{n}\left(a_{p q}^{R^{+}}+a_{p q}^{I^{+}}+a_{p q}^{J^{+}}+a_{p q}^{K^{+}}\right)\left(L_{f}^{R}\left|e_{q}^{R}(t)\right|\right. \\
&\left.+L_{f}^{I}\left|e_{q}^{I}(t)\right|+L_{f}^{J}\left|e_{q}^{J}(t)\right|+L_{f}^{K}\left|e_{q}^{K}(t)\right|\right)+\sum_{q=1}^{n}\left(b_{p q}^{R^{+}}+b_{p q}^{I^{+}}+b_{p q}^{J^{+}}\right. \\
&\left.+b_{p q}^{K^{+}}\right)\left(L_{g}^{R}\left|e_{q}^{R}\left(t-\tau_{p q}(t)\right)\right|+L_{g}^{I}\left|e_{q}^{I}\left(t-\tau_{p q}(t)\right)\right|+L_{g}^{J}\left|e_{q}^{J}\left(t-\tau_{p q}(t)\right)\right|\right. \\
&\left.+L_{g}^{K}\left|e_{q}^{K}\left(t-\tau_{p q}(t)\right)\right|\right)+\sum_{q=1}^{n}\left(p_{p q}^{R^{+}}+p_{p q}^{I^{+}}+p_{p q}^{J^{+}}+p_{p q}^{K^{+}}\right)\left(L_{h}^{R}\left|e_{q}^{R}(t)\right|\right. \\
&\left.+L_{h}^{I}\left|e_{q}^{I}(t)\right|+L_{h}^{J}\left|e_{q}^{J}(t)\right|+L_{h}^{K}\left|e_{q}^{K}(t)\right|\right)+\sum_{q=1}^{n}\left(q_{p q}^{R^{+}}+q_{p q}^{I^{+}}+q_{p q}^{J^{+}}\right. \\
&\left.+q_{p q}^{K^{+}}\right)\left(L_{\bar{h}}^{R}\left|e_{q}^{R}\left(t-\sigma_{p q}(t)\right)\right|+L_{\bar{h}}^{I}\left|e_{q}^{I}\left(t-\sigma_{p q}(t)\right)\right|\right. \\
&\left.+L_{\bar{h}}^{J}\left|e_{q}^{J}\left(t-\sigma_{p q}(t)\right)\right|+L_{\bar{h}}^{K}\left|e_{q}^{K}\left(t-\sigma_{p q}(t)\right)\right|\right) .
\end{aligned}
$$

Construct a Lyapunov function as follows:

$$
V(t)=V^{R}(t)+V^{I}(t)+V^{J}(t)+V^{K}(t),
$$

where

$$
\begin{aligned}
V^{l}(t)= & \sum_{p=1}^{n}\left(\left|e_{p}^{l}(t)\right| e^{\lambda t}+\Delta_{p}(t)\right), \quad l \in T, \\
\Delta_{p}(t)= & \frac{1}{\alpha} \sum_{q=1}^{n}\left(b_{p q}^{R^{+}}+b_{p q}^{I^{+}}+b_{p q}^{J^{+}}+b_{p q}^{K^{+}}\right) \int_{t-\tau_{p q}(t)}^{t}\left(L_{g}^{R}\left|e_{q}^{R}(s)\right|\right. \\
& \left.+L_{g}^{I}\left|e_{q}^{I}(s)\right|+L_{g}^{J}\left|e_{q}^{J}(s)\right|+L_{g}^{K}\left|e_{q}^{K}(s)\right|\right) e^{\lambda(s+\tau)} \mathrm{d} s \\
& +\frac{1}{\beta} \sum_{q=1}^{n}\left(q_{p q}^{R^{+}}+q_{p q}^{I^{+}}+q_{p q}^{J^{+}}+q_{p q}^{K^{+}}\right) \int_{t-\sigma_{p q}(t)}^{t}\left(L_{\bar{h}}^{R}\left|e_{q}^{R}(s)\right|\right. \\
& \left.+L_{\bar{h}}^{I}\left|e_{q}^{I}(s)\right|+L_{\bar{h}}^{J}\left|e_{q}^{J}(s)\right|+L_{\bar{h}}^{K}\left|e_{q}^{K}(s)\right|\right) e^{\lambda(s+\sigma)} \mathrm{d} s, \quad p \in S .
\end{aligned}
$$


Computing the upper right derivative of $V(t)$ along the solutions of (10), we have

$$
\begin{aligned}
& D^{+} V^{R}(t) \\
& \leq \sum_{p=1}^{n}\left\{\lambda e^{\lambda t}\left|e_{p}^{R}(t)\right|+e^{\lambda t} D^{+}\left|e_{p}^{R}(t)\right|+\frac{1}{\alpha} \sum_{q=1}^{n}\left(b_{p q}^{R^{+}}+b_{p q}^{I^{+}}+b_{p q}^{J^{+}}+b_{p q}^{K^{+}}\right)\right. \\
& \times\left(L_{g}^{R}\left|e_{q}^{R}(t)\right|+L_{g}^{I}\left|e_{q}^{I}(t)\right|+L_{g}^{J}\left|e_{q}^{J}(t)\right|+L_{g}^{K}\left|e_{q}^{K}(t)\right|\right) e^{\lambda(t+\tau)} \\
& -\frac{1}{\alpha} \sum_{q=1}^{n}\left(b_{p q}^{R^{+}}+b_{p q}^{I^{+}}+b_{p q}^{J^{+}}+b_{p q}^{K^{+}}\right)\left(L_{g}^{R}\left|e_{q}^{R}\left(t-\tau_{p q}(t)\right)\right|\right. \\
& \left.+L_{g}^{I}\left|e_{q}^{I}\left(t-\tau_{p q}(t)\right)\right|+L_{g}^{J}\left|e_{q}^{J}\left(t-\tau_{p q}(t)\right)\right|+L_{g}^{K}\left|e_{q}^{K}\left(t-\tau_{p q}(t)\right)\right|\right) \\
& \times e^{\lambda\left(t-\tau_{p q}(t)+\tau\right)}\left(1-\tau_{p q}^{\prime}(t)\right)+\frac{1}{\beta} \sum_{q=1}^{n}\left(q_{p q}^{R^{+}}+q_{p q}^{I^{+}}+q_{p q}^{J^{+}}+q_{p q}^{K^{+}}\right) \\
& \times\left(L_{\bar{h}}^{R}\left|e_{q}^{R}(t)\right|+L_{\bar{h}}^{I}\left|e_{q}^{I}(t)\right|+L_{\bar{h}}^{J}\left|e_{q}^{J}(t)\right|+L_{\bar{h}}^{K}\left|e_{q}^{K}(t)\right|\right) e^{\lambda(t+\sigma)} \\
& -\frac{1}{\beta} \sum_{q=1}^{n}\left(q_{p q}^{R^{+}}+q_{p q}^{I^{+}}+q_{p q}^{J^{+}}+q_{p q}^{K^{+}}\right)\left(L_{\bar{h}}^{R}\left|e_{q}^{R}\left(t-\sigma_{p q}(t)\right)\right|\right. \\
& +L_{\bar{h}}^{I}\left|e_{q}^{I}\left(t-\sigma_{p q}(t)\right)\right|+L_{\bar{h}}^{J}\left|e_{q}^{J}\left(t-\sigma_{p q}(t)\right)\right| \\
& \left.\left.+L_{\breve{h}}^{K}\left|e_{q}^{K}\left(t-\sigma_{p q}(t)\right)\right|\right) e^{\lambda\left(t-\sigma_{p q}(t)+\sigma\right)}\left(1-\sigma_{p q}^{\prime}(t)\right)\right\} \\
& \leq \sum_{p=1}^{n}\left\{\left(\lambda-c_{p}^{-}-d_{p}^{-}\right) e^{\lambda t}\left|e_{p}^{R}(t)\right|+\sum_{q=1}^{n}\left(a_{p q}^{R^{+}}+a_{p q}^{I^{+}}+a_{p q}^{J^{+}}+a_{p q}^{K^{+}}\right)\right. \\
& \times\left(L_{f}^{R}\left|e_{q}^{R}(t)\right|+L_{f}^{I}\left|e_{q}^{I}(t)\right|+L_{f}^{J}\left|e_{q}^{J}(t)\right|+L_{f}^{K}\left|e_{q}^{K}(t)\right|\right) e^{\lambda t} \\
& +\sum_{q=1}^{n}\left(b_{p q}^{R^{+}}+b_{p q}^{I^{+}}+b_{p q}^{J^{+}}+b_{p q}^{K^{+}}\right)\left(L_{g}^{R}\left|e_{q}^{R}\left(t-\tau_{p q}(t)\right)\right|\right. \\
& \left.+L_{g}^{I}\left|e_{q}^{I}\left(t-\tau_{p q}(t)\right)\right|+L_{g}^{J}\left|e_{q}^{J}\left(t-\tau_{p q}(t)\right)\right|+L_{g}^{K}\left|e_{q}^{K}\left(t-\tau_{p q}(t)\right)\right|\right) e^{\lambda t} \\
& +\sum_{q=1}^{n}\left(p_{p q}^{R^{+}}+p_{p q}^{I^{+}}+p_{p q}^{J^{+}}+p_{p q}^{K^{+}}\right)\left(L_{h}^{R}\left|e_{q}^{R}(t)\right|+L_{h}^{I}\left|e_{q}^{I}(t)\right|\right. \\
& \left.+L_{h}^{J}\left|e_{q}^{J}(t)\right|+L_{h}^{K}\left|e_{q}^{K}(t)\right|\right) e^{\lambda t}+\sum_{q=1}^{n}\left(q_{p q}^{R^{+}}+q_{p q}^{I^{+}}+q_{p q}^{I^{+}}+q_{p q}^{K^{+}}\right) \\
& \times\left(L_{\bar{h}}^{R}\left|e_{q}^{R}\left(t-\sigma_{p q}(t)\right)\right|+L_{\bar{h}}^{I}\left|e_{q}^{I}\left(t-\sigma_{p q}(t)\right)\right|+L_{\bar{h}}^{J}\left|e_{q}^{J}\left(t-\sigma_{p q}(t)\right)\right|\right. \\
& \left.+L_{\bar{h}}^{K}\left|e_{q}^{K}\left(t-\sigma_{p q}(t)\right)\right|\right) e^{\lambda t}+\frac{1}{\alpha} \sum_{q=1}^{n}\left(b_{p q}^{R^{+}}+b_{p q}^{I^{+}}+b_{p q}^{J^{+}}+b_{p q}^{K^{+}}\right) \\
& \times\left(L_{g}^{R}\left|e_{q}^{R}(t)\right|+L_{g}^{I}\left|e_{q}^{I}(t)\right|+L_{g}^{J}\left|e_{q}^{J}(t)\right|+L_{g}^{K}\left|e_{q}^{K}(t)\right|\right) e^{\lambda(t+\tau)} \\
& -\sum_{q=1}^{n}\left(b_{p q}^{R^{+}}+b_{p q}^{I^{+}}+b_{p q}^{J^{+}}+b_{p q}^{K^{+}}\right)\left(L_{g}^{R}\left|e_{q}^{R}\left(t-\tau_{p q}(t)\right)\right|\right. \\
& \left.+L_{g}^{I}\left|e_{q}^{I}\left(t-\tau_{p q}(t)\right)\right|+L_{g}^{J}\left|e_{q}^{J}\left(t-\tau_{p q}(t)\right)\right|+L_{g}^{K}\left|e_{q}^{K}\left(t-\tau_{p q}(t)\right)\right|\right) e^{\lambda t}
\end{aligned}
$$




$$
\begin{aligned}
& +\frac{1}{\beta} \sum_{q=1}^{n}\left(q_{p q}^{R^{+}}+q_{p q}^{I^{+}}+q_{p q}^{J^{+}}+q_{p q}^{K^{+}}\right)\left(L_{\bar{h}}^{R}\left|e_{q}^{R}(t)\right|+L_{\bar{h}}^{I}\left|e_{q}^{I}(t)\right|\right. \\
& \left.+L_{\bar{h}}^{J}\left|e_{q}^{J}(t)\right|+L_{\bar{h}}^{K}\left|e_{q}^{K}(t)\right|\right) e^{\lambda(t+\sigma)}-\sum_{q=1}^{n}\left(q_{p q}^{R^{+}}+q_{p q}^{I^{+}}+q_{p q}^{J^{+}}+q_{p q}^{K^{+}}\right) \\
& \times\left(L_{\bar{h}}^{R}\left|e_{q}^{R}\left(t-\sigma_{p q}(t)\right)\right|+L_{\bar{h}}^{I}\left|e_{q}^{I}\left(t-\sigma_{p q}(t)\right)\right|+L_{\bar{h}}^{J}\left|e_{q}^{J}\left(t-\sigma_{p q}(t)\right)\right|\right. \\
& \left.\left.+L_{\bar{h}}^{K}\left|e_{q}^{K}\left(t-\sigma_{p q}(t)\right)\right|\right) e^{\lambda t}\right\} \\
& \leq \sum_{p=1}^{n}\left\{\left(\lambda-c_{p}^{-}-d_{p}^{-}\right)\left|e_{p}^{R}(t)\right|+\sum_{q=1}^{n}\left(a_{p q}^{R^{+}}+a_{p q}^{I^{+}}+a_{p q}^{J^{+}}+a_{p q}^{K^{+}}\right)\right. \\
& \times\left(L_{f}^{R}\left|e_{q}^{R}(t)\right|+L_{f}^{I}\left|e_{q}^{I}(t)\right|+L_{f}^{J}\left|e_{q}^{J}(t)\right|+L_{f}^{K}\left|e_{q}^{K}(t)\right|\right)+\frac{1}{\alpha} \sum_{q=1}^{n}\left(b_{p q}^{R^{+}}\right. \\
& \left.+b_{p q}^{I^{+}}+b_{p q}^{J^{+}}+b_{p q}^{K^{+}}\right)\left(L_{g}^{R}\left|e_{q}^{R}(t)\right|+L_{g}^{I}\left|e_{q}^{I}(t)\right|+L_{g}^{J}\left|e_{q}^{J}(t)\right|\right. \\
& \left.+L_{g}^{K}\left|e_{q}^{K}(t)\right|\right) e^{\lambda \tau}+\sum_{q=1}^{n}\left(p_{p q}^{R^{+}}+p_{p q}^{I^{+}}+p_{p q}^{J^{+}}+p_{p q}^{K^{+}}\right)\left(L_{h}^{R}\left|e_{q}^{R}(t)\right|\right. \\
& \left.+L_{h}^{I}\left|e_{q}^{I}(t)\right|+L_{h}^{J}\left|e_{q}^{J}(t)\right|+L_{h}^{K}\left|e_{q}^{K}(t)\right|\right) \\
& +\frac{1}{\beta} \sum_{q=1}^{n}\left(q_{p q}^{R^{+}}+q_{p q}^{I^{+}}+q_{p q}^{I^{+}}+q_{p q}^{K^{+}}\right)\left(L_{\bar{h}}^{R}\left|e_{q}^{R}(t)\right|+L_{\bar{h}}^{I}\left|e_{q}^{I}(t)\right|\right. \\
& \left.\left.+L_{\bar{h}}^{J}\left|e_{q}^{J}(t)\right|+L_{\bar{h}}^{K}\left|e_{q}^{K}(t)\right|\right) e^{\lambda \sigma}\right\} e^{\lambda t} \\
& \leq \sum_{p=1}^{n}\left\{\left(\lambda-c_{p}^{-}-d_{p}^{-}\right)+\sum_{q=1}^{n}\left(a_{p q}^{R^{+}}+a_{p q}^{I^{+}}+a_{p q}^{J^{+}}+a_{p q}^{K^{+}}\right)\left(L_{f}^{R}+L_{f}^{I}\right.\right. \\
& \left.+L_{f}^{J}+L_{f}^{K}\right)+\frac{1}{\alpha} \sum_{q=1}^{n}\left(b_{p q}^{R^{+}}+b_{p q}^{I^{+}}+b_{p q}^{J^{+}}+b_{p q}^{K^{+}}\right)\left(L_{g}^{R}+L_{g}^{I}\right. \\
& \left.+L_{g}^{I}+L_{g}^{K}\right) e^{\lambda \tau}+\sum_{q=1}^{n}\left(p_{p q}^{R^{+}}+p_{p q}^{I^{+}}+p_{p q}^{J^{+}}+p_{p q}^{K^{+}}\right)\left(L_{h}^{R}+L_{h}^{I}\right. \\
& \left.+L_{h}^{J}+L_{h}^{K}\right)+\frac{1}{\beta} \sum_{q=1}^{n}\left(q_{p q}^{R^{+}}+q_{p q}^{I^{+}}+q_{p q}^{J^{+}}+q_{p q}^{K^{+}}\right)\left(L_{\bar{h}}^{R}+L_{\bar{h}}^{I}\right. \\
& \left.\left.+L_{\bar{h}}^{J}+L_{\bar{h}}^{K}\right) e^{\lambda \sigma}\right\} e^{\lambda t}\|e(t)\| \\
& =\sum_{p=1}^{n}\left\{\left(\lambda-c_{p}^{-}-d_{p}^{-}\right)+A_{p}+\frac{1}{\alpha} B_{p} e^{\lambda \tau}+P_{p}+\frac{1}{\beta} Q_{p} e^{\lambda \sigma}\right\} e^{\lambda t}\|e(t)\| .
\end{aligned}
$$

Performing a similar calculation, we can obtain

$$
\begin{aligned}
D^{+} V^{l}(t) \leq & \sum_{p=1}^{n}\left\{\left(\lambda-c_{p}^{-}-d_{p}^{-}\right)+A_{p}+\frac{1}{\alpha} B_{p} e^{\lambda \tau}+P_{p}\right. \\
& \left.+\frac{1}{\beta} Q_{p} e^{\lambda \sigma}\right\} e^{\lambda t}\|e(t)\|, \quad l=I, J, K .
\end{aligned}
$$


In view of $\left(H_{6}\right),(12)$ and (13), we have

$$
D^{+} V(t) \leq 0 \text {. }
$$

Hence, $V(t) \leq V(0)$ for all $t \geq 0$.

On the other hand, we have

$$
\begin{aligned}
& V^{R}(0)=\sum_{p=1}^{n}\left\{\left|e_{p}^{R}(0)\right|+\frac{1}{\alpha} \sum_{q=1}^{n}\left(b_{p q}^{R^{+}}+b_{p q}^{I^{+}}+b_{p q}^{J^{+}}+b_{p q}^{K^{+}}\right)\right. \\
& \times \int_{0-\tau_{p q}(0)}^{0}\left(L_{g}^{R}\left|e_{q}^{R}(s)\right|+L_{g}^{I}\left|e_{q}^{I}(s)\right|+L_{g}^{J}\left|e_{q}^{J}(s)\right|\right. \\
& \left.+L_{g}^{K}\left|e_{q}^{K}(s)\right|\right) e^{\lambda(s+\tau)} \mathrm{d} s+\frac{1}{\beta} \sum_{q=1}^{n}\left(q_{p q}^{R^{+}}+q_{p q}^{I^{+}}+q_{p q}^{J^{+}}\right. \\
& \left.+q_{p q}^{K^{+}}\right) \int_{0-\sigma_{p q}(0)}^{0}\left(L_{\bar{h}}^{R}\left|e_{q}^{R}(s)\right|+L_{\bar{h}}^{I}\left|e_{q}^{I}(s)\right|\right. \\
& \left.\left.+L_{\bar{h}}^{J}\left|e_{q}^{J}(s)\right|+L_{\bar{h}}^{K}\left|e_{q}^{K}(s)\right|\right) e^{\lambda(s+\sigma)} \mathrm{d} s\right\} \\
& \leq \sum_{p=1}^{n}\left\{1+\frac{1}{\alpha} \sum_{q=1}^{n}\left(b_{p q}^{R^{+}}+b_{p q}^{I^{+}}+b_{p q}^{J^{+}}+b_{p q}^{K^{+}}\right)\left(L_{g}^{R}+L_{g}^{I}\right.\right. \\
& \left.+L_{g}^{J}+L_{g}^{K}\right) \frac{\left(e^{\lambda \tau}-1\right)}{\lambda}+\frac{1}{\beta} \sum_{q=1}^{n}\left(q_{p q}^{R^{+}}+q_{p q}^{I^{+}}+q_{p q}^{J^{+}}+q_{p q}^{K^{+}}\right) \\
& \left.\times\left(L_{\bar{h}}^{R}+L_{\bar{h}}^{I}+L_{\bar{h}}^{J}+L_{\bar{h}}^{K}\right) \frac{\left(e^{\lambda \sigma}-1\right)}{\lambda}\right\}\|\psi-\varphi\|_{0} \\
& =\sum_{p=1}^{n}\left\{1+\frac{\left(e^{\lambda \tau}-1\right)}{\alpha \lambda} B_{p}+\frac{\left(e^{\lambda \sigma}-1\right)}{\beta \lambda} Q_{p}\right\}\|\psi-\varphi\|_{0} .
\end{aligned}
$$

Similarly, we can get

$$
V^{l}(0) \leq \sum_{p=1}^{n}\left\{1+\frac{\left(e^{\lambda \tau}-1\right)}{\alpha \lambda} B_{p}+\frac{\left(e^{\lambda \sigma}-1\right)}{\beta \lambda} Q_{p}\right\}\|\psi-\varphi\|_{0}, \quad l=I, J, K .
$$

It is obvious that

$$
\|y(t)-x(t)\| e^{\lambda t}=\|e(t)\| e^{\lambda t} \leq V(t), \quad t \geq 0
$$

Hence, we have

$$
\|y(t)-x(t)\| \leq V(t) e^{-\lambda t} \leq V(0) e^{-\lambda t} \leq M\|\psi-\varphi\|_{0} e^{-\lambda t}, \quad t \geq 0,
$$

where

$$
M=\sum_{p=1}^{n}\left\{1+\frac{\left(e^{\lambda \tau}-1\right)}{\alpha \lambda} B_{p}+\frac{\left(e^{\lambda \sigma}-1\right)}{\beta \lambda} Q_{p}\right\}>1 .
$$


Therefore, system (1) and system (9) are globally exponentially synchronized. This completes the proof.

\section{A numerical example}

In this section, we give two numerical examples to illustrate the effectiveness of our results.

Example 1 Consider the following drive system:

$$
x_{p}^{\prime}(t)=-c_{p}(t) x_{p}(t)+\sum_{q=1}^{2} a_{p q}(t) f_{q}\left(x_{q}(t)\right)+\sum_{q=1}^{2} b_{p q}(t) g_{q}\left(x_{q}\left(t-\tau_{p q}(t)\right)\right)+J_{p}(t)
$$

where $p=1,2, v(t)=e^{|t|}$, and the coefficients are as follows:

$$
\begin{aligned}
& f_{q}\left(x_{q}\right)=\frac{\left|x^{R}+1\right|-\left|x^{R}-1\right|}{4}+\frac{1}{2} k \sin \left(x^{I}+x^{J}+x^{K}\right), \\
& g_{q}\left(x_{q}\right)=\frac{1}{4} \arctan x^{R}+\frac{1}{4} i \sin x^{K}+\frac{1}{4} j \tanh \left(x_{q}^{I}+x_{q}^{J}\right), \\
& c_{1}(t)=4+|\sin (\sqrt{5} t)+\cos t|, \quad c_{2}(t)=7-2 \cos \sqrt{2} t, \quad \tau_{p q}(t)=\frac{1}{2}(1+\sin 2 t), \\
& a_{11}(t)=a_{12}(t)=0.2 \sin (\sqrt{2} t)+0.1 i(\sin (\sqrt{2} t)+\cos t)+0.1 k \cos (\sqrt{7} t), \\
& a_{21}(t)=a_{22}(t)=0.1 \sin (\sqrt{5} t)+0.3 j \sin t+0.2 k(\sin t+\cos \sqrt{3} t), \\
& b_{11}(t)=b_{12}(t)=0.5 \cos (\sqrt{7} t)+0.4 k(\cos (\sqrt{3} t)+\sin \sqrt{2}(t)), \\
& b_{21}(t)=b_{22}(t)=0.3+0.4 i \sin (\sqrt{3} t)+0.3 j \sin \sqrt{2} t+0.1 k, \\
& J_{1}(t)=2(\sin t+\cos (2 t))+i 2 \sin (\sqrt{5} t)+j 2 \cos (\sqrt{7} t)+k\left(1.9 \cos \sqrt{3} t+0.1 e^{-t}\right), \\
& J_{2}(t)=1.9 \cos \sqrt{3} t+0.1 e^{-t}+i 2 \sin t+j\left(1.9 \cos t+0.1 e^{-t}\right)+k 2(\sin (\sqrt{3} t)+\sin t) .
\end{aligned}
$$

By a simple computation, for $p=1,2, l \in T$, we have

$$
\begin{aligned}
& c_{1}^{-}=4, \quad c_{2}^{-}=5, \quad J_{p}^{l^{+}}=2, \quad L_{f}^{l}=\frac{1}{2}, \quad L_{g}^{l}=\frac{1}{4}, \\
& A_{1}=1.6, \quad B_{1}=1.8, \quad A_{2}=2.4, \quad B_{2}=2.2,
\end{aligned}
$$

and

$$
\begin{aligned}
& \kappa=\max \left\{\frac{J_{1}^{l^{+}}}{c_{1}^{-}}, \frac{J_{2}^{l^{+}}}{c_{2}^{-}}\right\}=\frac{1}{2}, \\
& \rho=\max \left\{\frac{1}{c_{1}^{-}}\left(A_{1}+B_{1}\right), \frac{1}{c_{2}^{-}}\left(A_{2}+B_{2}\right)\right\}=\max \{0.85,0.92\}=0.92<1 .
\end{aligned}
$$

So, all the assumptions of Theorem 1 is satisfied. Therefore, by Theorem 1, we see that (14) has a unique weighted pseudo-almost periodic solution (see Fig. 1). 

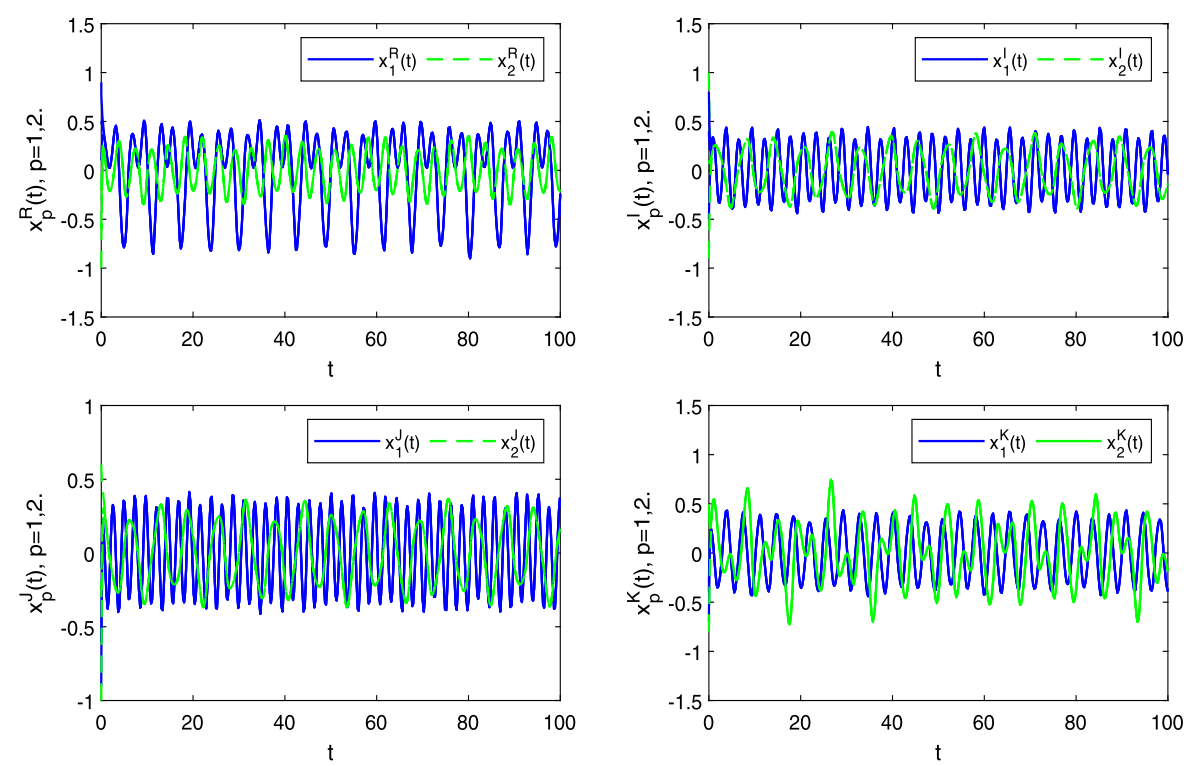

Figure 1 The states of four parts of $x_{1}(t)$ and $x_{2}(t)$

Example 2 Consider the following drive system:

$$
x_{p}^{\prime}(t)=-c_{p}(t) x_{p}(t)+\sum_{q=1}^{2} a_{p q}(t) f_{q}\left(x_{q}(t)\right)+\sum_{q=1}^{2} b_{p q}(t) g_{q}\left(x_{q}\left(t-\tau_{p q}(t)\right)\right)+J_{p}(t)
$$

and the response system

$$
\begin{aligned}
y_{p}^{\prime}(t)= & -c_{p}(t) y_{p}(t)+\sum_{q=1}^{2} a_{p q}(t) f_{q}\left(y_{q}(t)\right) \\
& +\sum_{q=1}^{2} b_{p q}(t) g_{q}\left(y_{q}\left(t-\tau_{p q}(t)\right)\right)+J_{p}(t)+u_{p}(t),
\end{aligned}
$$

where $p=1,2$,

$$
u_{p}(t)=-d_{p}(t) e_{p}(t)+\sum_{q=1}^{2} p_{p q}(t) h_{q}\left(e_{q}(t)\right)+\sum_{q=1}^{2} q_{p q}(t) \bar{h}_{q}\left(e_{q}\left(t-\sigma_{p q}(t)\right)\right) .
$$

Consider the weight $v(t)=e^{|t|}$ and the coefficients are taken as follows:

$$
\begin{aligned}
& f_{q}\left(x_{q}\right)=\frac{1}{5} \tanh x_{q}^{R}+\frac{1}{5} i\left|x_{q}^{I}+x_{q}^{J}+x_{q}^{K}\right|+j \frac{1}{7} \sin x_{q}^{J}+\frac{1}{5} k\left|x_{q}^{K}\right|, \\
& g_{q}\left(x_{q}\right)=\frac{1}{7} \sin \left(x_{q}^{R}+\frac{1}{4} x_{q}^{I}\right)+\frac{1}{7} i\left|x_{q}^{J}+x_{q}^{K}\right|+\frac{1}{9} j \tanh x_{q}^{K}+\frac{1}{7} k \sin x_{q}^{I}, \\
& h_{q}\left(e_{q}\right)=\frac{1}{5} \tanh e_{q}^{R}+\frac{1}{5} i\left|e_{q}^{R}+e_{q}^{I}+e_{q}^{J}+e_{q}^{K}\right|+\frac{1}{8} j \sin ^{2} e_{q}^{K}+\frac{1}{5} k \sin e_{q}^{I}, \\
& \bar{h}_{q}\left(e_{q}\right)=\frac{1}{7} \sin \left(e_{q}^{I}+e_{q}^{J}\right)+\frac{1}{7} i\left|e_{q}^{J}+e_{q}^{K}\right|+\frac{1}{8} j \tanh \left(e_{q}^{R}+e_{q}^{K}\right)+\frac{1}{7} k \sin ^{2} e_{q}^{I},
\end{aligned}
$$




$$
\begin{aligned}
& a_{11}(t)=0.1(\cos (2 t)+i \sin (2 t)+j+k \sin (\sqrt{3} t)), \\
& a_{12}(t)=0.1(\sin (\sqrt{2} t)+\cos (2 t)+j \sin t+k \cos (\sqrt{3} t)), \\
& a_{21}(t)=0.1(\cos (\sqrt{2} t)+i(\sin (\sqrt{3} t)+\cos t)), \\
& a_{22}(t)=0.1(\sin t+i \cos (\sqrt{3} t)+k \sin (\sqrt{2} t)), \\
& b_{11}(t)=0.1\left(\sin t+\cos (\sqrt{2} t)+j \cos ^{2} t+k \cos (2 t)\right) \text {, } \\
& b_{12}(t)=0.1(\sin (\sqrt{3} t)+k(\cos (2 t)+\sin \sqrt{3} t)) \text {, } \\
& b_{21}(t)=0.1(\sin (2 t)+j \sin (\sqrt{5} t)+k \cos (3 t)) \text {, } \\
& b_{22}(t)=0.1(i \cos t+j \sin t+k \sin (\sqrt{3} t)) \text {, } \\
& p_{11}(t)=0.1+i 0.1 \cos t+k\left(0.09 \cos t+0.01 e^{-t}\right), \\
& p_{12}(t)=0.1 \cos (\sqrt{3} t)+i\left(0.09 \sin t+0.01 e^{-t}\right)+j \cos ^{2} t, \\
& p_{21}(t)=0.09 \sin (\sqrt{2} t)+0.01 e^{-t}+j 0.1 \cos t+0.1 k, \\
& p_{22}(t)=i 0.1 \sin (3 t)+k\left(0.09(\sin (\sqrt{3} t)+\cos (2 t))+0.01 e^{-t}\right), \\
& q_{11}(t)=i 0.1 \sin \sqrt{3} t+j 0.1 \cos \sqrt{2} t+k\left(0.09+0.01 e^{-t}\right), \\
& \left.q_{12}(t)=0.1 \cos (2 t)+i\left(0.09 \cos t+0.01 e^{-t}\right)+j 0.1 \sin ^{2} t\right), \\
& q_{21}(t)=0.1(\sin (\sqrt{2})+\cos (4 t))+k 0.1 \sin t, \\
& q_{22}(t)=0.09 \cos (\sqrt{2} t)+0.01 e^{-t}+i 0.1 \sin \sqrt{2} t+k 0.1 \sin (3 t), \\
& c_{1}(t)=2 \sin (\sqrt{2} t)+4, \quad c_{2}(t)=5-2 \cos (\sqrt{3} t), \\
& d_{1}(t)=(\sin (\sqrt{2} t)+\cos t)+5, \quad d_{2}(t)=6-2 \cos (\sqrt{5} t), \\
& J_{1}(t)=0.9 \sin t+0.1 e^{-t}+i \cos (\sqrt{3} t)+j \sin (\sqrt{3} t)+k \cos (2 t), \\
& J_{2}(t)=\sin t+i(\sin t+\cos (\sqrt{2} t))+j\left(0.9 \cos t+0.1 e^{-t}\right)+k \sin (2 t), \\
& \tau_{11}(t)=\frac{1}{5}, \quad \tau_{12}(t)=\frac{1}{4}|\sin (2 t)|, \quad \tau_{21}(t)=\frac{1}{6}|\cos (2 t)|, \quad \tau_{22}(t)=\frac{1}{4}, \\
& \sigma_{11}(t)=\frac{1}{9}|\cos t|, \quad \sigma_{12}(t)=\frac{1}{8}, \quad \sigma_{21}(t)=\frac{1}{10}|\sin (2 t)|, \quad \sigma_{22}(t)=\frac{1}{8}|\sin (2 t)| .
\end{aligned}
$$

By a simple computation, for $p=1,2, l \in T$, we have

$$
\begin{aligned}
& c_{1}^{-}=2, \quad c_{2}^{-}=3, \quad d_{1}^{-}=d_{2}^{-}=4, \quad \tau=\frac{1}{4}, \\
& \sigma=\frac{1}{8}, \quad J_{p}^{l^{+}}=1, \quad \alpha=\frac{3}{4}, \quad \beta=\frac{7}{8}, \\
& L_{f}^{l}=L_{h}^{l}=\frac{1}{5}, \quad L_{g}^{l}=L_{\bar{h}}^{l}=\frac{1}{7}, \quad A_{1}=1.12, \quad B_{1} \approx 0.5714, \quad P_{1}=0.96, \\
& Q_{1} \approx 0.6857, \quad A_{2} \approx 0.6857, \quad B_{2} \approx 0.6857, \quad P_{2} \approx 0.5714, \quad Q_{2} \approx 0.5714,
\end{aligned}
$$

and

$$
\kappa=\max \left\{\frac{J_{1}^{l^{+}}}{c_{1}^{-}}, \frac{J_{2}^{l^{+}}}{c_{2}^{-}}\right\}=\frac{1}{2},
$$



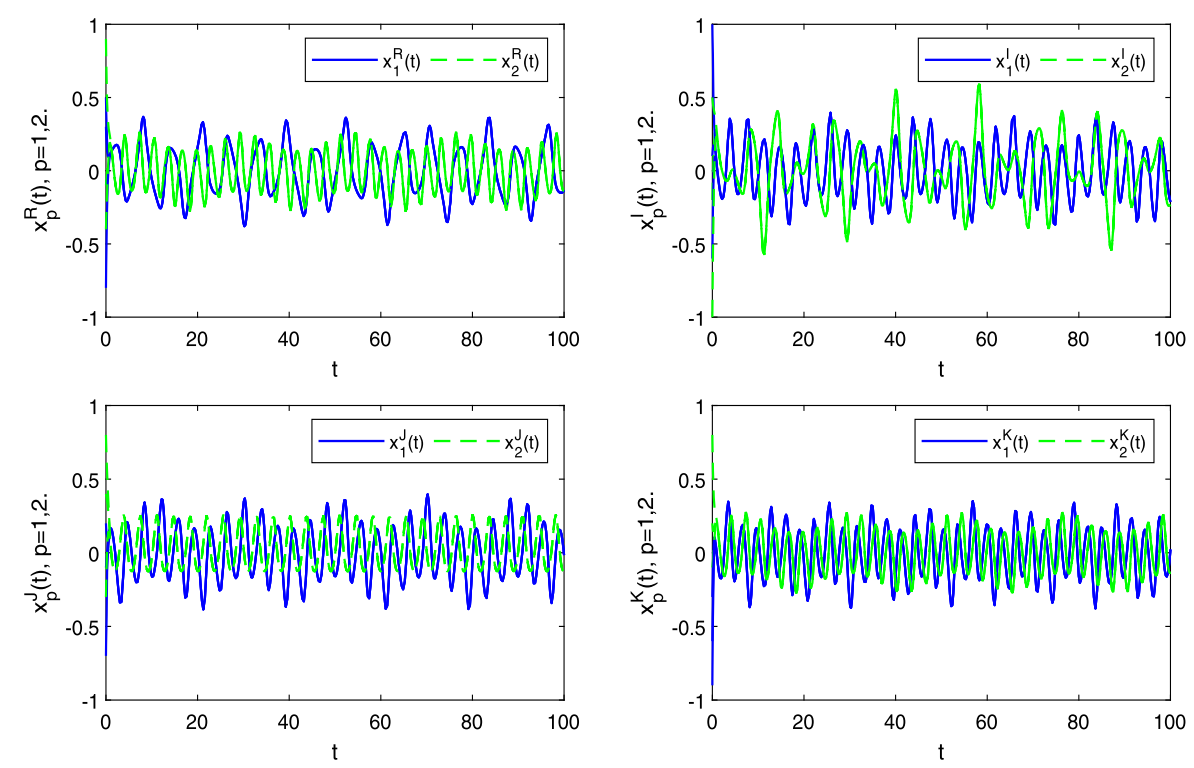

Figure 2 The states of four parts of $x_{1}(t)$ and $x_{2}(t)$
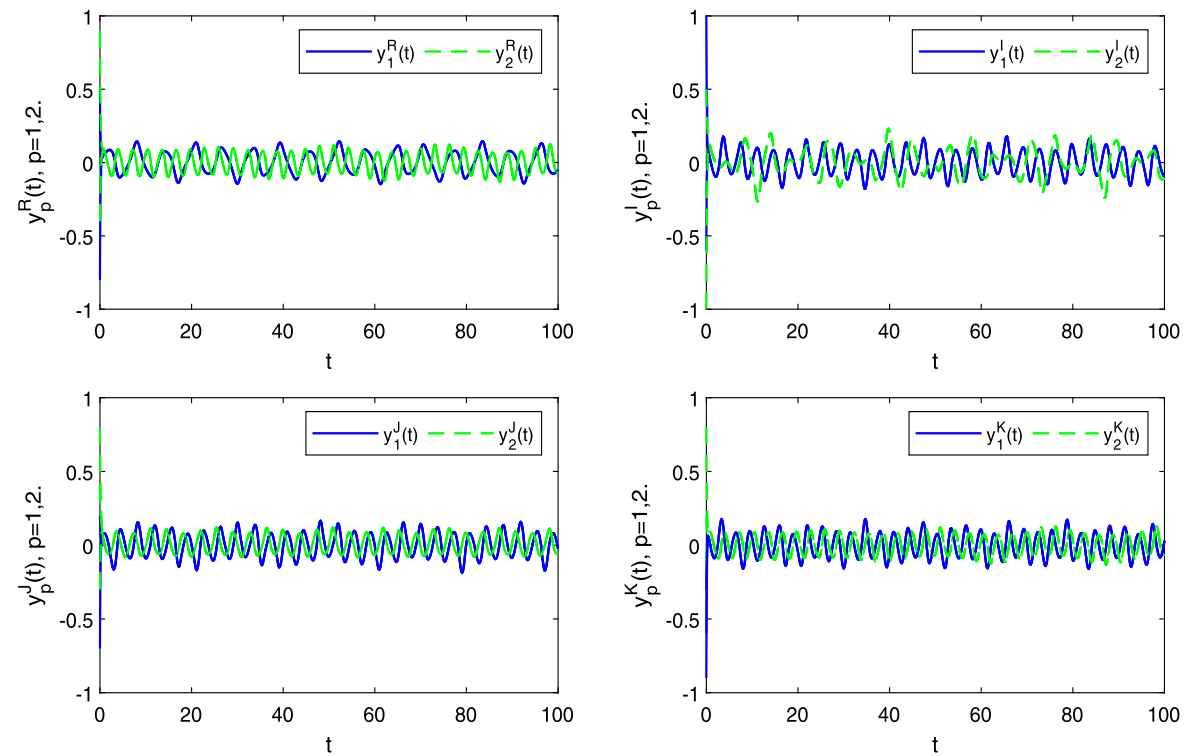

Figure 3 The states of four parts of $y_{1}(t)$ and $y_{2}(t)$

$$
\rho=\max \left\{\frac{1}{c_{1}^{-}}\left(A_{1}+B_{1}\right), \frac{1}{c_{2}^{-}}\left(A_{2}+B_{2}\right)\right\}=\max \{0.8457,0.4571\}=0.8457<1 .
$$

Take $\lambda=1$, then we have

$$
\begin{aligned}
& \left(\lambda-c_{1}^{-}-d_{1}^{-}\right)+A_{1}+\frac{1}{\alpha} B_{1} e^{\lambda \tau}+P_{1}+\frac{1}{\beta} Q_{1} e^{\lambda \sigma} \approx-1.05374<0, \\
& \left(\lambda-c_{2}^{-}-d_{2}^{-}\right)+A_{2}+\frac{1}{\alpha} B_{2} e^{\lambda \tau}+P_{2}+\frac{1}{\beta} Q_{2} e^{\lambda \sigma} \approx-2.82898<0 .
\end{aligned}
$$




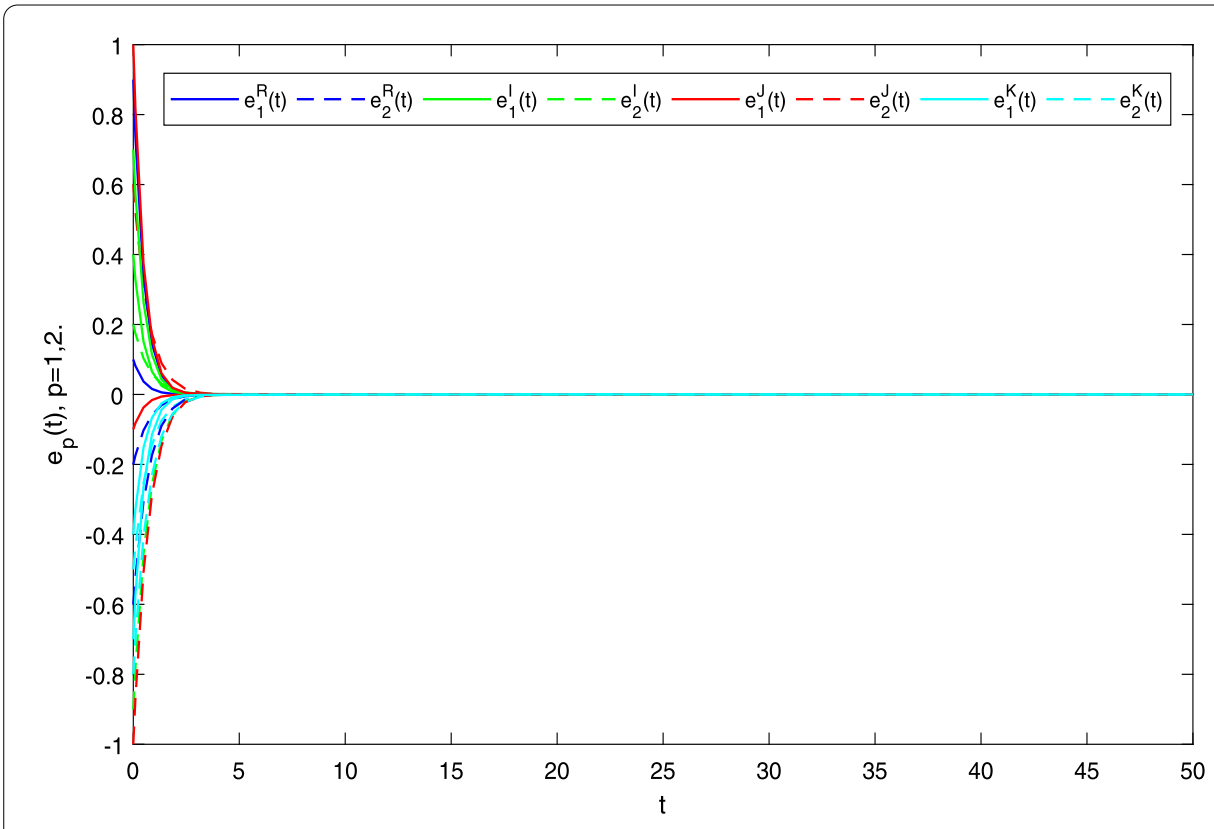

Figure 4 Synchronization

So, all the assumptions of Theorem 2 are satisfied. So by Theorem 2, system (15) has a unique weighted pseudo-almost periodic solution and system (15) and (16) are globally exponentially synchronized (see Figs. 2-4).

\section{Conclusion}

In this work, we studied the existence of weighted pseudo-almost periodic solutions of delayed QVCNNs. Moreover, when the drive system has a unique weighted pseudoalmost periodic solution, we investigated global exponential synchronization of the driveresponse structure of delayed QVCNNs with weighted pseudo-almost periodic coefficients. The approach of this paper can be used to study the problem of the weighted pseudo-almost periodic solutions and synchronization for other types of neural networks.

Acknowledgements

Not applicable.

Funding

This work is supported by the National Natural Sciences Foundation of People's Republic of China under Grant No. 11861072.

\section{Availability of data and materials}

Data sharing not applicable to this article as no datasets were generated or analysed during the current study.

Ethics approval and consent to participate

Not applicable.

\section{Competing interests}

The authors declare that they have no competing interests.

Consent for publication

Not applicable. 


\section{Publisher's Note}

Springer Nature remains neutral with regard to jurisdictional claims in published maps and institutional affiliations.

\section{Received: 15 February 2019 Accepted: 19 August 2019 Published online: 29 August 2019}

\section{References}

1. Chua, L.O., Yang, L.: Cellular neural networks: theory. IEEE Trans. Circuits Syst. 35(10), 1257-1272 (1988)

2. Chua, L.O., Yang, L.: Cellular neural networks: applications. IEEE Trans. Circuits Syst. 35(10), 1273-1290 (1988)

3. Gilli, M., Biey, M., Checco, P.: Equilibrium analysis of cellular neural networks. IEEE Trans. Circuits Syst. I, Regul. Pap. 51(5), 903-912 (2004)

4. Zhang, J., Gui, Z.: Periodic solutions of nonautonomous cellular neural networks with impulses and delays. Nonlinear Anal., Real World Appl. 10(3), 1891-1903 (2009)

5. Li, Y., Zhao, L., Chen, X.: Existence of periodic solutions for neutral type cellular neural networks with delays. Appl. Math. Model. 36(3), 1173-1183 (2012)

6. Li, Y., Wang, C.: Almost periodic solutions of shunting inhibitory cellular neural networks on time scales. Commun. Nonlinear Sci. Numer. Simul. 17(8), 3258-3266 (2012)

7. Hunang, Z:: Almost periodic solutions for fuzzy cellular neural networks with multi-proportional delays. Int. J. Mach. Learn. Cybern. 8(4), 1323-1331 (2017)

8. Zhang, A.: Pseudo almost periodic solutions for CNNs with oscillating leakage coefficients and complex deviating arguments. J. Exp. Theor. Artif. Intell. 30(1), 89-100 (2018)

9. Liu, B., Tunç, C.: Pseudo almost periodic solutions for CNNs with leakage delays and complex deviating arguments. Neural Comput. Appl. 26(2), 429-435 (2015)

10. M'Hamdi, M.S., Aouiti, C., Touati, A., Alimi, A.M., Sansel, V.: Weighted pseudo almost-periodic solutions of shunting inhibitory cellular neural networks with mixed delays. Acta Math. Sci. 36(6), 1662-1682 (2016)

11. Xu, Y.: Exponential stability of weighted pseudo almost periodic solutions for HCNNs with mixed delays. Neural Process. Lett. 46(2), 507-519 (2017)

12. Duan, L., Fang, X., Huang, C.: Global exponential convergence in a delayed almost periodic Nicholson's blowflies model with discontinuous harvesting. Math. Methods Appl. Sci. 41(5), 1954-1965 (2017)

13. Duan, L., Huang, L., Guo, Z., Fang, X.: Periodic attractor for reaction-diffusion high-order Hopfield neural networks with time-varying delays. Comput. Math. Appl. 73(2), 233-245 (2017)

14. Huang, C., Liu, B., Tian, X., Yang, L., Zhang, X.: Global convergence on asymptotically almost periodic SICNNs with nonlinear decay functions. Neural Process. Lett. 49(2), 625-641 (2019)

15. Huang, C., Zhang, H., Huang, L.: Almost periodicity analysis for a delayed Nicholson's blowflies model with nonlinear density-dependent mortality term. Commun. Pure Appl. Anal. 18(6), 3337-3349 (2019)

16. Li, Y., Qin, J., Li, B.: Existence and global exponential stability of anti-periodic solutions for delayed quaternion-valued cellular neural networks with impulsive effects. Math. Methods Appl. Sci. 42(1), 5-23 (2019)

17. Huang, C., Zhang, H.: Periodicity of non-autonomous inertial neural networks involving proportional delays and non-reduced order method. Int. J. Biomath. 12(02), 1950016 (2019)

18. Huang, C., Cao, J., Wen, F., Yang, X.: Stability analysis of SIR model with distributed delay on complex networks. PLoS One 11(8), 0158813 (2016)

19. Li, Y., Qin, J., Li, B.: Periodic solutions for quaternion-valued fuzzy cellular neural networks with time-varying delays. Adv. Differ. Equ. 2019(63), (2019)

20. Wang, J., Chen, X., Huang, L.: The number and stability of limit cycles for planar piecewise linear systems of node-saddle type. J. Math. Anal. Appl. 469(1), 405-427 (2019)

21. Cai, Z., Huang, J., Huang, L.: Periodic orbit analysis for the delayed Filippov system. Proc. Am. Math. Soc. 146(11), 4667-4682 (2018)

22. Huang, C., Liu, B.: New studies on dynamic analysis of inertial neural networks involving non-reduced order method. Neurocomputing 325, 283-287 (2019)

23. Liu, B., Tunç, C.: Pseudo almost periodic solutions for a class of nonlinear Duffing system with a deviating argument. J. Appl. Math. Comput. 49(1-2), 233-242 (2015)

24. Liu, B., Tunç, C.: Pseudo almost periodic solutions for a class of first order differential iterative equations. Appl. Math Lett. 40, 29-34 (2015)

25. Pecora, L.M., Carroll, T.L.: Synchronization in chaotic systems. Phys. Rev. Lett. 64(8), $821-824$ (1990)

26. Xia, Y., Yang, Z., Han, M.: Synchronization schemes for coupled identical Yang-Yang type fuzzy cellular neural networks. Commun. Nonlinear Sci. Numer. Simul. 14(9-10), 3645-3659 (2009)

27. Chen, J., Zeng, Z., Jiang, P.: Global Mittag-Leffler stability and synchronization of memristor-based fractional-order neural networks. Neural Netw. 51, 1-8 (2014)

28. Zhou, Y., Li, C., Huang, T., Wang, X.: Impulsive stabilization and synchronization of Hopfield-type neural networks with impulse time window. Neural Comput. Appl. 28(4), 775-782 (2017)

29. Xing, J., Jiang, H., Hu, C.: Exponential synchronization for delayed recurrent neural networks via periodically intermittent control. Neurocomputing 113, 122-129 (2013)

30. Xing, Z., Peng, J., Wang, K.: Exponential synchronization of chaotic neural networks with time delays: a M-matrix approach. Nonlinear Dyn. 62(4), 867-874 (2010)

31. Tu, Z., Ding, N., Li, L., Feng, Y., Zou, L., Zhang, W.: Adaptive synchronization of memristive neural networks with time-varying delays and reaction-diffusion term. Appl. Math. Comput. 311, 118-128 (2017)

32. Cai, Z., Huang, L., Guo, Z., Zhang, L., Wan, X.: Periodic synchronization control of discontinuous delayed networks by using extended Filippov-framework. Neural Netw. 68, 96-110 (2015)

33. Cai, Z., Huang, L., Wang, D., Zhang, L.: Periodic synchronization in delayed memristive neural networks based on Filippov systems. J. Franklin Inst. 352(10), 4638-4663 (2015)

34. Yang, C., Huang, L., Li, F.: Exponential synchronization control of discontinuous nonautonomous networks and autonomous coupled networks. Complexity 2018, 1-10 (2018)

35. Sudbery, A.: Quaternionic analysis. Math. Proc. Camb. Philos. Soc. 85(2), 199-225 (1979) 
36. Isokawa, T., Kusakabe, T., Matsui, N., Peper, F.: Quaternion neural network and its application. In: Knowledge-Based Intelligent Information and Engineering Systems, pp. 318-324. Springer, Berlin (2003)

37. Matsui, N., Isokawa, T., Kusamichi, H., Peper, F., Nishimura, H.: Quaternion neural network with geometrical operators. J. Intell. Fuzzy Syst. 15, 149-164 (2004)

38. Chen, X., Li, Z., Song, Q., Hu, J., Tan, Y.: Robust stability analysis of quaternion-valued neural networks with time delays and parameter uncertainties. Neural Netw. 91, 55-65 (2017)

39. Meng, X., Li, Y.: Pseudo almost periodic solutions for quaternion-valued cellular neural networks with discrete and distributed delays. J. Inequal. Appl. 2018(245), (2018)

40. Tu, Z., Cao, J., Alsaedi, A., Hayat, T.: Global dissipativity analysis for delayed quaternion-valued neural networks. Neural Netw. 89, 97-104 (2017)

41. Shu, H., Song, Q., Liu, Y., Zhao, Z., Alsaadi, F.E.: Global $\mu$-stability of quaternion-valued neural networks with non-differentiable time-varying delays. Neurocomputing 247, 202-212 (2017)

42. Li, Y., Qin, J.: Existence and global exponential stability of periodic solutions for quaternion-valued cellular neural networks with time-varying delays. Neurocomputing 2018, 91-103 (2018)

43. Li, Y., Meng, X.: Existence and global exponential stability of pseudo almost periodic solutions for neutral type quaternion-valued neural networks with delays in the leakage term on time scales. Complexity 2017(10), 1-15 (2017)

44. Fink, A.M.: Almost Periodic Differential Equations. Springer, Berlin (1974)

45. Zhang, C.: Almost Periodic Type Functions and Ergodicity. Science Press, Beijing (2003)

46. Diagana, T: Almost Automorphic Type and Almost Periodic Type Functions in Abstract Spaces. Springer, Berlin (2013)

47. Li, Y., Zhao, L.: Weighted pseudo-almost periodic functions on time scales with applications to cellular neural networks with discrete delays. Math. Methods Appl. Sci. 40(6), 1905-1921 (2017)

\section{Submit your manuscript to a SpringerOpen ${ }^{\circ}$ journal and benefit from:}

- Convenient online submission

- Rigorous peer review

- Open access: articles freely available online

- High visibility within the field

- Retaining the copyright to your article

Submit your next manuscript at $\gg$ springeropen.com 